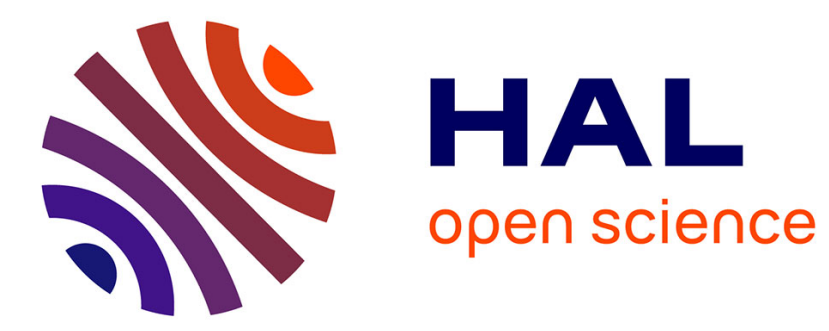

\title{
On the Expressiveness of Symmetric Communication
}

Thomas Given-Wilson, Axel Legay

\section{To cite this version:}

Thomas Given-Wilson, Axel Legay. On the Expressiveness of Symmetric Communication. Theoretical Aspects of Computing - ICTAC 2016, Oct 2016, Taipei, Taiwan. pp.139-157, 10.1007/978-3-31946750-4_9. hal-01241839v3

\section{HAL Id: hal-01241839 \\ https://hal.inria.fr/hal-01241839v3}

Submitted on 17 Jul 2016

HAL is a multi-disciplinary open access archive for the deposit and dissemination of scientific research documents, whether they are published or not. The documents may come from teaching and research institutions in France or abroad, or from public or private research centers.
L'archive ouverte pluridisciplinaire $\mathbf{H A L}$, est destinée au dépôt et à la diffusion de documents scientifiques de niveau recherche, publiés ou non, émanant des établissements d'enseignement et de recherche français ou étrangers, des laboratoires publics ou privés. 


\title{
On the Expressiveness of Symmetric Communication
}

\author{
Thomas Given-Wilson and Axel Legay
}

\begin{abstract}
Inria
Abstract. The expressiveness of communication primitives has been explored in a common framework based on the $\pi$-calculus by considering four features: synchronism, arity, communication medium, and pattern-matching. These all assume asymmetric communication between input and output primitives, however some calculi consider more symmetric approaches to communication such as fusion calculus and Concurrent Pattern Calculus. Symmetry can be considered either as supporting exchange of information between an action and co-action, or as unification of actions. By means of possibility/impossibility of encodings, this paper shows that the exchange approach is related to, or more expressive than, many previously considered languages. Meanwhile, the unification approach is more expressive than some, but mostly unrelated to, other languages.
\end{abstract}

\section{Introduction}

The expressiveness of process calculi based upon their communication primitives has been widely explored before $[7,26,4,16,10,12]$. In $[16,12]$ this is detailed by examining combinations of four features, namely: synchronism, arity, communication medium, and pattern-matching. These features are able to represent many popular calculi including: monadic or polyadic $\pi$-calculus [24, 23]; LINDA [9]; asymmetric variations of Concurrent Pattern Calculus (CPC) [14, 10,11]; and Psi calculi [1]. However, all these calculi exploit upon asymmetric input and output behaviour.

Symmetric behaviour has been considered before in process calculi. One example, fusion calculus [28] shifts away from explicit input and output of names to instead fuse them together in a symmetric equivalence relation. Another is CPC that shifted away from input and output primitives to a single primitive that can do both input or output (and equality tests) via the unification of patterns [14].

This paper abstracts away from specific calculi in the style of $[16,12]$ to provide a general account of the expressiveness of symmetric communication primitives. Here symmetric communication does not require that input or output be associated to a particular action or co-action primitive, indeed all communication primitives can perform all possible input, output, or equality tests. This captures the spirit of both fusion calculus and CPC's interaction paradigms, while also generalising to something that can be applied to any calculus. However, there is some complexity when deciding how this should be represented in an abstract calculus since there are two reasonable choices. The first choice is to consider symmetry to support exchange, where an action and co-action interact and allow both input and output from either side. This exchange approach of action and co-action with both input and output on both sides aligns with the fusion calculus style of interaction. The second choice is to consider symmetry to support symmetric unification, where a single communication primitive is used for interaction. This approach of a symmetric unification via a single interaction primitive and allowing self recognition (as well as exchange) aligns with CPC style interaction. 
The solution here is to consider both; leading to the symmetry feature having three possible instantiations. Asymmetric where there is explicit input (that can only contain input patterns) and output (that can only contain output terms), e.g.

$$
n(\lambda x, \lambda y) . P|\bar{n}\langle a, b\rangle \cdot Q \quad \longmapsto \quad\{a / x, b / y\} P| Q .
$$

Exchange where there are two explicit primitives action and co-action that can mix input patterns and output terms, e.g.

$$
n(\lambda x, b) \cdot P|\bar{n}\langle a, \lambda y\rangle \cdot Q \quad \longmapsto \quad\{a / x\} P|\{b / y\} Q .
$$

Unification where this is a single communication primitive that contains a single class of patterns that unify with one-another, e.g.

$$
n(\lambda x \bullet b \bullet c) . P|n(a \bullet \lambda y \bullet c) . Q \quad \longmapsto \quad\{a / x\} P|\{b / y\} Q .
$$

By extending prior work with symmetry (and removing synchronism since all exchange and unification languages must be synchronous), the original twelve calculi of [12] are here expanded to thirty-six. This paper details the relations between the original twelve calculi and the twenty-four new calculi, yielding the following key results.

In general exchange languages are more expressive than their asymmetric counterparts. However, there are methods to encode exchange languages with bounded matching capabilities (i.e. a finite limit to the number of names that can be matched) into asymmetric languages. Thus indicating that pattern-matching is highly significant as a factor for determining encodings.

Within the exchange languages, expressiveness increases in a similar manner as the asymmetric languages. The exceptions occur when pattern-matching is intensional, since polyadic exchange languages cannot be encoded into monadic languages, but polyadic asymmetric languages can be encoded into monadic intensional languages.

No unification language can be encoded into an exchange or asymmetric language, this is due to a self recognising process $S$ that can reduce with itself but not alone something that cannot be defined in any asymmetric or exchange language.

Unification languages do not require name-matching to be able to encode namematching languages, thus no-matching unification languages can encode name-matching asymmetric and exchange languages. An interesting result, since no asymmetric or exchange language without (at least) name-matching can encode name-matching.

Within the unification languages, relations between languages are identical to the asymmetric setting. This indicates that although unification is a different approach to interaction, the other features are largely unaffected by changing the interaction setting.

The structure of the paper is as follows. Section 2 introduces the considered calculi. Section 3 revises the encoding criteria used for comparing calculi. Section 4 provides a diagrammatic overview of the results. Section 5 explores new relations concerning asymmetric and exchange languages. Section 6 considers unification languages and their relations. Section 7 concludes, and discusses choices made here $\&$ in related work.

\section{Calculi}

This section defines the syntax, operational, and behavioural semantics of the calculi considered here. This relies heavily on the well-known notions developed for the $\pi$ - 
calculus, the reference framework, and adapts them when necessary. With the exception of the symmetric constructs this is similar to prior definitions from [12].

Assume a countable set of names $\mathcal{N}($ denoted $a, b, c)$. Name-matching patterns (denoted $m, n, o$ ), and symmetric patterns (denoted $p, q$ ) are defined by:

$$
\begin{aligned}
& m, n::=\lambda x \text { binding name } \quad p, q::=a \text { name } \\
& \mid\ulcorner a\urcorner \text { name-match } \quad \mid m \quad \text { name-match patterns } \\
& \mid p \bullet q \quad \text { compound. }
\end{aligned}
$$

Binding names (denoted $\lambda x, \lambda y, \lambda z$ ) are used to indicate input behaviour, name-matches $\ulcorner a\urcorner$ test for equality, and compounds combine two symmetric patterns into one (all as in $[14,12])$. The free names $f n(\cdot)$, binding names $b n(\cdot)$, and matched names $m n(\cdot)$ of namematching and symmetric patterns are as expected, taking the union of sub-patterns for compound patterns. A symmetric pattern is linear iff all binding names within the pattern are pairwise distinct. The rest of this paper will only consider linear input patterns.

The symmetric patterns are chosen here to be very general and capture many concepts, however to clearly define the languages in this paper, define the following. The terms (denoted $s, t$ ) are the symmetric patterns that contain no binding names or namematches. (These correspond to the terms of [12], the communicable patterns of CPC, and the output structures of Psi calculi.) The intensional patterns (denoted $f, g$ ) are the symmetric patterns that contain no names, i.e. they consist entirely of name-matching patterns and compounds. (These correspond to the intensional patterns of [12].)

The (parametric) syntax for the languages is:

$$
P, Q, R::=\mathbf{0} \mid \text { ACT.P | COACT.P }|(v n) P| P|Q| \text { if } s=t \text { then } P \text { else } Q|* P| \sqrt{ } \text {. }
$$

The different languages are obtained by replacing the action $A C T$ and co-action $C O A C T$ with the various definitions. The rest of the process forms as are usual: $\mathbf{0}$ denotes the null process; restriction $(v n) P$ restricts the visibility of $n$ to $P$; and parallel composition $P \mid Q$ allows independent evolution of $P$ and $Q$. The if $s=t$ then $P$ else $Q$ represents conditional equivalence with if $s=t$ then $P$ used when $Q$ is $\mathbf{0}$ (like the name match of $\pi$-calculus, if $s=t$ then $P$ else $Q$ blocks either $P$ when $s \neq t$ or $Q$ when $s=t$ ). The $* P$ represents replication of the process $P$. Finally, the $\sqrt{ }$ is used to represent a success process or state, exploited for reasoning about encodings as in $[18,10]$.

This paper considers the possible combinations of four features for communication: arity (monadic vs polyadic data), communication medium (message passing vs shared dataspaces), pattern-matching (simple binding vs name equality vs intensionality), and symmetry (asymmetric vs exchange vs unification). As a result there exist thirty-six languages denoted by $\mathcal{L}_{\alpha, \beta, \gamma, \delta}$ where:

$\alpha=M$ for monadic data, and $P$ for polyadic data.

$\beta=D$ for dataspace-based communication, and $C$ for channel-based communications.

$\gamma=N O$ for no matching capability, $N M$ for name-matching, and $I$ for intensionality.

$\delta=A$ for asymmetric communication, $E$ for exchange communication, and $U$ for unification communication.

For simplicity a dash - is used when the instantiation of that feature is unimportant. 


$$
\begin{aligned}
& \mathcal{L}_{-,-, N O,-}: I N::=\lambda x \quad \text { OUT }::=a \quad A L L::=\lambda x \mid a \quad C H::=a \\
& \mathcal{L}_{-,-, N M,-}: I N::=m \quad \text { OUT }::=a \quad \text { ALL }::=m \mid a \quad C H::=a \\
& \mathcal{L}_{-,-, I,-}: I N::=f \quad \text { OUT }::=t \quad \text { ALL }::=p \quad C H::=t \\
& \mathcal{L}_{M, D,-, A}: A C T::=(I N) \quad C O A C T::=\langle O U T\rangle \\
& \mathcal{L}_{M, C,-, A}: A C T::=C H(I N) \quad C O A C T::=\overline{C H}\langle O U T\rangle \\
& \mathcal{L}_{P, D,-, A}: A C T::=(\widetilde{I N}) \quad C O A C T::=\langle\widetilde{O U T}\rangle \\
& \mathcal{L}_{P, C,-, A}: A C T::=C H(\widetilde{I N}) \quad \text { COACT }::=\overline{C H}\langle\widetilde{O U T}\rangle \\
& \mathcal{L}_{M, D,-, E}: A C T::=(A L L) \quad C O A C T::=\langle A L L\rangle \\
& \mathcal{L}_{M, C,-, E}: A C T::=C H(A L L) \quad C O A C T::=\overline{C H}\langle A L L\rangle \\
& \mathcal{L}_{P, D,-, E}: A C T::=(\widetilde{A L L}) \quad C O A C T::=\langle\widetilde{A L L}\rangle \\
& \mathcal{L}_{P, C,-, E}: A C T::=C H(\widetilde{A L L}) \\
& \mathcal{L}_{M, D,-, U}: A C T, C O A C T::=(A L L) \\
& \mathcal{L}_{M, C,-, U}: A C T, C O A C T::=C H(A L L) \\
& \mathcal{L}_{P, D,-, U}: A C T, C O A C T::=(\widetilde{A L L}) \\
& \mathcal{L}_{P, C,-, U}: A C T, C O A C T::=C H(\widetilde{A L L}) . \\
& \text { COACT ::= } \overline{C H}\langle\widetilde{A L L}\rangle
\end{aligned}
$$

Fig. 1. Languages in this paper.

Thus the syntax of every language is obtained from the productions in Figure 1. The first three lines define the components of communication primitives based upon the pattern-matching of the language; with input patterns IN, output patterns OUT, combined patterns $A L L$, and channel structures $C H$. The rest defines the languages by their action $A C T$ and co-action $C O A C T$ using the communication primitives. Here the denotation $\widetilde{r}$ represents a sequence of the form $\cdot_{1}, \cdot_{2}, \ldots,{ }_{n}$ and can be used for names, binding names, terms, and both kinds of patterns. As usual $(v x) P$ and binding names $\lambda x$ in any form (including $I N$ and $A L L$ ) bind $x$ in $P$. The corresponding notions of free and bound names of a process, denoted $\mathrm{fn}(P)$ and $\mathrm{bn}(P)$, are as usual. An action or co-action is linear if all binding names occur exactly once; this paper shall only consider linear actions and co-actions.

Observe that: monadic languages have a single $I N, O U T$, or $A L L$ in their action and co-action, while polyadic languages have sequences. Dataspace-based languages are distinct from channel-based languages by not having a channel $\mathrm{CH}$ that is used for interaction. No-matching languages allow only binding names in $I N$, name-matching languages also allow name-matches, and intensional languages allow intensional patterns in $I N$. No-matching and name-matching languages only allow names in $O U T$, while intensional languages allow terms. Lastly, asymmetric languages only allow IN in actions and $O U T$ in co-actions, while exchange and unification languages allow $A L L$ in both (the latter by defining the co-action to be the action).

Note that $\alpha$-conversion (denoted $=_{\alpha}$ ) is assumed in the usual manner. Finally, the structural equivalence relation $\equiv$ is defined as follows:

$$
\begin{array}{lrrrr}
P|Q \equiv Q| P & P|(Q \mid R) \equiv(P \mid Q)| R & P \equiv P^{\prime} & \text { if } P={ }_{\alpha} P^{\prime} & P \mid \mathbf{0} \equiv P \\
(v a) \mathbf{0} \equiv \mathbf{0} & (v a)(v b) P \equiv(v b)(v a) P & P \mid(v a) Q \equiv(v a)(P \mid Q) & \text { if } a \notin \operatorname{fn}(P) .
\end{array}
$$

Most of the asymmetric languages correspond to the communication paradigm of popular process calculi, including (but not limited to): monadic or polyadic $\pi$-calculus; LINDA; asymmetric variations of CPC; and Psi calculi. For details on these and other 
languages see $[16,12]$. With respect to symmetry: $\mathcal{L}_{P, C, N O, E}$ is closest in communication paradigm to the fusion calculus [28] although the scope of binding in communication is different. $\mathcal{L}_{M, D, I, U}$ corresponds to the communication paradigm of CPC; and $\mathcal{L}_{M, D, I, E}$, $\mathcal{L}_{M, C, I, E}$, and $\mathcal{L}_{M, C, I, U}$ to the communication paradigms of variants of CPC [10].

Remark 1. Most of the languages can be ordered; in particular $\mathcal{L}_{\alpha_{1}, \beta_{1}, \gamma_{1}, \delta_{1}}$ is a sublanguage of $\mathcal{L}_{\alpha_{2}, \beta_{2}, \gamma_{2}, \delta_{2}}$ if it holds that $\alpha_{1} \leq \alpha_{2}$ and $\beta_{1} \leq \beta_{2}$ and $\gamma_{1} \leq \gamma_{2}$ and $\delta_{1} \leq \delta_{2}$, where $\leq$ is the least reflexive relation satisfying the following axioms:

$$
M \leq P \quad D \leq C \quad N O \leq N M \leq I \quad A \leq E .
$$

This can be understood as a limited language variation being a special case of a more general language. Monadic communication is polyadic communication with all tuples of arity one. Dataspace-based communication is channel-based communication with all $k$-ary tuples communicating with channel name $k$. All name-matching communication is intensional communication without any compounds, and no-matching capability communication is both without any compounds and with only names or only binding names in patterns. Asymmetric communication is exchange with only input patterns in actions, and only output patterns in co-actions; and exchange languages are unification languages with restrictions upon the unification (this does not induce $\leq$, see Section 6).

The operational semantics of the languages is given here via reductions as in [23, 12]. An alternative style is via a labelled transition system (LTS) such as [16]. Here the reduction based style is chosen for simplicity. The LTS style can be used for intensional and symmetric languages [1,10], and indeed captures many of the languages here [13].

Substitutions, denoted $\sigma, \rho$ in non-pattern-matching and name-matching languages are mappings (with finite domain) from names to names. For intensional languages substitutions are mappings from names to terms. The application of a substitution $\sigma$ to a pattern $p$ is defined as follows:

$$
\sigma x=\left\{\begin{array}{ll}
\sigma(x) x \in \operatorname{domain}(\sigma) \\
x x \notin \operatorname{domain}(\sigma)
\end{array} \quad \sigma\ulcorner x\urcorner=\ulcorner(\sigma x)\urcorner \quad \sigma(p \bullet q)=(\sigma p) \bullet(\sigma q) .\right.
$$

Where substitution is as usual on names, and on the understanding that the name-match syntax can be applied to any term by defining: $\ulcorner(s \bullet t) \stackrel{\text { def }}{=}\ulcorner s\urcorner \bullet \tau$. Given a substitution $\sigma$ and a process $P$, denote with $\sigma P$ the usual capture-avoiding application of $\sigma$ to $P$. As usual capture can be avoided by exploiting $\alpha$-equivalence [2]

Interaction between processes is handled by unification of patterns with other patterns. The core unification can be used for all languages as defined by the unify rule $\{p \| q\}$ of a single pattern $p$ and a single pattern $q$ to create two substitutions $\sigma$ and $\rho$ whose domains are the binding names of $p$ and $q$, respectively. This is defined by:

$$
\begin{array}{rlrl}
\{a \| a\}=\{a \|\ulcorner a\urcorner\}=\{\ulcorner a\urcorner \| a\}=\{\ulcorner a\urcorner \|\ulcorner\urcorner\}) & \stackrel{\text { def }}{=}(\{\},\{\}) \\
\{\lambda x \| t\} \stackrel{\text { def }}{=}(\{t / x\},\{\}) & \text { if } t \text { is a term } \\
\{s \| \lambda x\} \stackrel{\text { def }}{=}(\{\},\{s / x\}) & \text { if } s \text { is a term } \\
\left\{p_{1} \bullet p_{2} \| q_{1} \bullet q_{2}\right\} \stackrel{\text { def }}{=}\left(\sigma_{1} \cup \sigma_{2}, \rho_{1} \cup \rho_{2}\right) & \text { if }\left\{p_{i} \| q_{i}\right\}=\left(\sigma_{i}, \rho_{i}\right) \text { for } i \in\{1,2\} \\
\{p \| q\} & \text { undefined } & \text { otherwise. }
\end{array}
$$


Names and name-matches unify if they are for the same name. A binding name unifies with a term to produce a binding of the name to that term. Two compounds unify if their components unify; the resulting substitutions are the unions of those produced by unifying the components. Otherwise the unification is undefined (impossible). Note that the substitutions being combined have disjoint domain due to linearity of patterns, and this holds for the following two rules also.

The asymmetric and exchange languages exploit the poly-match rule $\left.\mathrm{MATcH}_{\mathbf{p}} ; \widetilde{q}\right)$ that determines the matches of two sequences of patterns $\widetilde{p}$ and $\widetilde{q}$ to produce a pair of substitutions, as defined below:

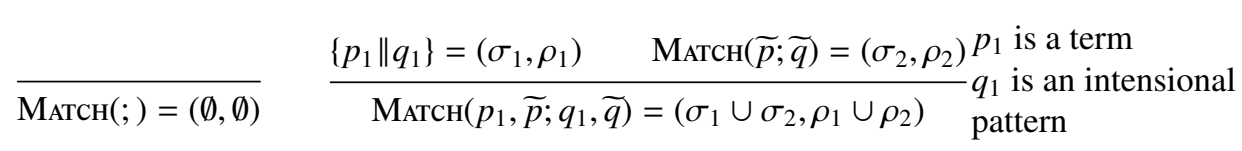

$$
\begin{aligned}
& \begin{array}{ll}
\left\{p_{1} \| q_{1}\right\}=\left(\sigma_{1}, \rho_{1}\right) \quad \operatorname{Match}(\widetilde{p} ; \widetilde{q})=\left(\sigma_{2}, \rho_{2}\right) & p_{1} \text { is an intensional } \\
\operatorname{Match}\left(p_{1}, \widetilde{p} ; q_{1}, \widetilde{q}\right)=\left(\sigma_{1} \cup \sigma_{2}, \rho_{1} \cup \rho_{2}\right) & \text { pattern } \\
q_{1} \text { is a term. }
\end{array}
\end{aligned}
$$

The empty sequence matches with the empty sequence to produce empty substitutions. Otherwise when there are sequences $p_{1}, \widetilde{p}$ and $q_{1}, \widetilde{q}$ where $p_{1}$ is a term and $q_{1}$ is an intensional pattern (or vice versa) then they are unified $\left\{p_{1} \| q_{1}\right\}$ and the remaining sequences use the poly-match rule. If both are defined and yield substitutions, the union of substitutions is yielded. Otherwise the poly-match is undefined, such as when; when a single unification fails, a term is aligned with a term, an intensional pattern with an intensional pattern, or when the sequences are of unequal arity.

The unification languages use the poly-unify rule $\operatorname{UnIFY}(\widetilde{p} ; \widetilde{q})$ that is the same as the poly-match rule (without the side conditions) as shown below:

$$
\operatorname{UnIFY}(;)=(\emptyset, \emptyset) \frac{\left\{p_{1} \| q_{1}\right\}=\left(\sigma_{1}, \rho_{1}\right) \quad \operatorname{UnIFY}(\widetilde{p} ; \widetilde{q})=\left(\sigma_{2}, \rho_{2}\right)}{\operatorname{UnIFy}\left(p_{1}, \widetilde{p} ; q_{1}, \widetilde{q}\right)=\left(\sigma_{1} \cup \sigma_{2}, \rho_{1} \cup \rho_{2}\right)} .
$$

Interaction is now defined by the following two axioms. The first

$$
\bar{s}\langle\widetilde{p}\rangle . P|s(\widetilde{q}) \cdot Q \longmapsto(\sigma P)|(\rho Q) \quad \text { Матсн }(\widetilde{p} ; \widetilde{q})=(\sigma, \rho)
$$

for asymmetric and exchange languages; and the second

$$
s(\widetilde{p}) \cdot P|s(\widetilde{q}) \cdot Q \longmapsto(\sigma P)|(\rho Q) \quad \operatorname{UNIFy}(\widetilde{p} ; \widetilde{q})=(\sigma, \rho)
$$

for the unification languages. In both the $s$ 's are omitted for dataspace-based languages. Both axioms state that when the the symmetric patterns $\widetilde{p}$ and $\widetilde{q}$ poly-match or polyunify, respectively, (and in the channel-based setting the input and output are along the same channel) to yield the substitutions $\sigma$ and $\rho$, they reduce to $\sigma$ applied to $P$ in parallel with $\rho$ applied to $Q$.

The reduction relation $\longmapsto$ also includes the following:

$$
\begin{aligned}
& \frac{P \longmapsto P^{\prime}}{P\left|Q \longmapsto P^{\prime}\right| Q} \quad \frac{P \longmapsto P^{\prime}}{(v a) P \longmapsto(v a) P^{\prime}} \quad \frac{P \equiv Q \quad Q \longmapsto Q^{\prime} \quad Q^{\prime} \equiv P^{\prime}}{P \longmapsto P^{\prime}} \\
& s=t \quad P|Q \longmapsto S \quad s \neq t \quad P| R \longmapsto S \\
& \overline{P \mid \text { if } s=t \text { then } Q \text { else } R \longmapsto S} \quad \overline{P \mid \text { if } s=t \text { then } Q \text { else } R \longmapsto S}
\end{aligned}
$$


with $\Longleftrightarrow$ denoting the reflexive, transitive closure of $\longmapsto$.

Lastly, for each language let $\cong$ denote a reduction-sensitive reference behavioural equivalence for that language, e.g. a barbed equivalence. That is, a behavioural equivalence $\cong$ such that whenever $P \cong P^{\prime}$ and $P^{\prime} \longmapsto$ imply $P \longmapsto$ as in Definition 5.3 of [18] (observe that his rules out weak bisimulations for example). For the asymmetric languages these are already known, either by their equivalent language in the literature or from $[16,13,12]$. For the non-asymmetric languages the results in [13] can be applied.

\section{Encodings}

This section recalls the definition of valid encodings for formally relating process calculi (details in [18]) . The choice of valid encodings here is to align with prior works $[16,18,12]$ and where possible reuse prior results. These valid encodings are those used, sometimes with mild adaptations, in $[18,17,25,10,14]$ and have also inspired similar works $[21,22,31]$. However, there are alternative approaches to encoding criteria or comparing expressive power [30,3,7,5,27,31]. Further discussion of the choices of encodings, and contrasting with other approaches can be found in $[18,17,29,31,14]$.

An encoding of a language $\mathcal{L}_{1}$ into another language $\mathcal{L}_{2}$ is a pair $\left(\llbracket \cdot \rrbracket, \varphi_{\llbracket \rrbracket}\right)$ where $\llbracket \cdot \rrbracket$ translates every $\mathcal{L}_{1}$-process into an $\mathcal{L}_{2}$-process and $\varphi_{\| \rrbracket}$ maps every name (of the source language) into a tuple of $k$ names (of the target language), for $k>0$. In doing this, the translation may fix some names to play a precise rôle or may translate a single name into a tuple of names, this can be obtained by exploiting $\varphi_{\|\mathbb{I}\|}$.

Now consider only encodings that satisfy the following properties. Let a $k$-ary context $\left(\cdot_{1} ; \ldots ; \cdot{ }_{k}\right)$ be a process with $k$ holes. Denote with $\longmapsto^{\omega}$ an infinite sequence of reductions and let $P \Downarrow$ mean there exists $P^{\prime}$ such that $P \Longleftrightarrow P^{\prime}$ and $P^{\prime} \equiv P^{\prime \prime} \mid \sqrt{ }$ for some $P^{\prime \prime}$. Moreover, let $\cong$ denote the reference behavioural equivalence. Finally, to simplify reading, let $S$ range over processes of the source language (viz., $\mathcal{L}_{1}$ ) and $T$ range over processes of the target language (viz., $\mathcal{L}_{2}$ ).

Definition 1 (Valid Encoding). An encoding ([I. $\left.\rrbracket, \varphi_{\| \Perp}\right)$ of $\mathcal{L}_{1}$ into $\mathcal{L}_{2}$ is valid if it satisfies the following five properties:

1. Compositionality: for every $k$-ary operator op of $\mathcal{L}_{1}$ and for every subset of names $N$, there exists a $k$-ary context $C_{\mathrm{op}}^{N}\left(\cdot_{1} ; \ldots ;{ }_{k}\right)$ of $\mathcal{L}_{2}$ such that, for all $S_{1}, \ldots, S_{k}$ with $\mathrm{fn}\left(S_{1}, \ldots, S_{k}\right)=N$, it holds that $\llbracket \mathrm{op}\left(S_{1}, \ldots, S_{k}\right) \rrbracket=C_{\mathrm{op}}^{N}\left(\llbracket S_{1} \rrbracket ; \ldots ; \llbracket S_{k} \rrbracket\right)$.

2. Name invariance: for every $S$ and substitution $\sigma$, it holds that $\llbracket \sigma S \rrbracket=\sigma^{\prime} \llbracket S \rrbracket$ if $\sigma$ is injective and $\llbracket \sigma S \rrbracket \cong_{2} \quad \sigma^{\prime} \llbracket S \rrbracket$ otherwise where $\sigma^{\prime}$ is such that $\varphi_{\llbracket \rrbracket}(\sigma(a))=$ $\sigma^{\prime}\left(\varphi_{\llbracket \rrbracket}(a)\right)$ for every name a.

3. Operational correspondence:

- for all $S \varliminf_{1} S^{\prime}$, it holds that $\llbracket S \rrbracket \varliminf_{2} \cong_{2} \llbracket\left[S^{\prime} \rrbracket\right.$;

- for all $\llbracket S \rrbracket \longmapsto_{2} T$, there exists $S^{\prime}$ such that $S \longmapsto_{1} S^{\prime}$ and $T \varliminf_{2} \cong_{2} \llbracket S^{\prime} \rrbracket$.

4. Divergence reflection: for every $S$ such that $\llbracket S \rrbracket \longmapsto_{2}^{\omega}$, it holds that $S \longmapsto_{1}^{\omega}$.

5. Success sensitiveness: for every $S$, it holds that $S \Downarrow_{1}$ if and only if $\llbracket S \rrbracket \Downarrow_{2}$.

Proposition 1. Let $\llbracket \cdot \rrbracket$ be a valid encoding from $\mathcal{L}_{1}$ into $\mathcal{L}_{2}$; if there exist two $\mathcal{L}_{1}$ processes $P$ of the form $p_{1}\left(p_{2}\right) P^{\prime}$ and $Q$ of the form either $q_{1}\left(q_{2}\right) Q^{\prime}$ or $\overline{q_{1}}\left\langle q_{2}\right\rangle Q^{\prime}$ such that $P \mid Q \longmapsto$, then $\llbracket P \mid Q \rrbracket \longmapsto$. 
The following result exploits the matching degree of a language $\operatorname{MD}(\cdot)$, defined as the least upper bound on the number of names that can be matched to yield reduction.

Proposition 2 (Theorem 5.9 from [18]). If $\operatorname{MD}\left(\mathcal{L}_{1}\right)>\operatorname{MD}\left(\mathcal{L}_{2}\right)$ then there is no valid encoding of $\mathcal{L}_{1}$ into $\mathcal{L}_{2}$.

Proposition 3 (Theorem 5.8 from [18]). Assume there exists a $\mathcal{L}_{1}$-process $S$ such that $S \mapsto{ }_{1}$ and $S \Downarrow$ and $S \mid S \Downarrow$; moreover assume that every $\mathcal{L}_{2}$-process $T$ that does not reduce is such that $T \mid T \mapsto \mapsto_{2}$. Then there exists no valid encoding $\mathbb{\|} \cdot \rrbracket$ from $\mathcal{L}_{1}$ to $\mathcal{L}_{2}$.

The general way to prove the lack of a valid encoding is done as follows. By contradiction assuming there is a valid encoding $\llbracket \cdot \rrbracket$. Find a pair of processes $P$ and $Q$ that satisfy Proposition 1 such that $P \mid Q \longmapsto$ and $\llbracket P \mid Q \rrbracket \longmapsto$. From $Q$ obtain some $Q^{\prime}$ such that $P \mid Q^{\prime} \mapsto$ and $\llbracket P \mid Q^{\prime} \rrbracket \longmapsto$. Conclude by showing this in contradiction with some properties of the encoding or one of the propositions above.

The following result is a consequence of

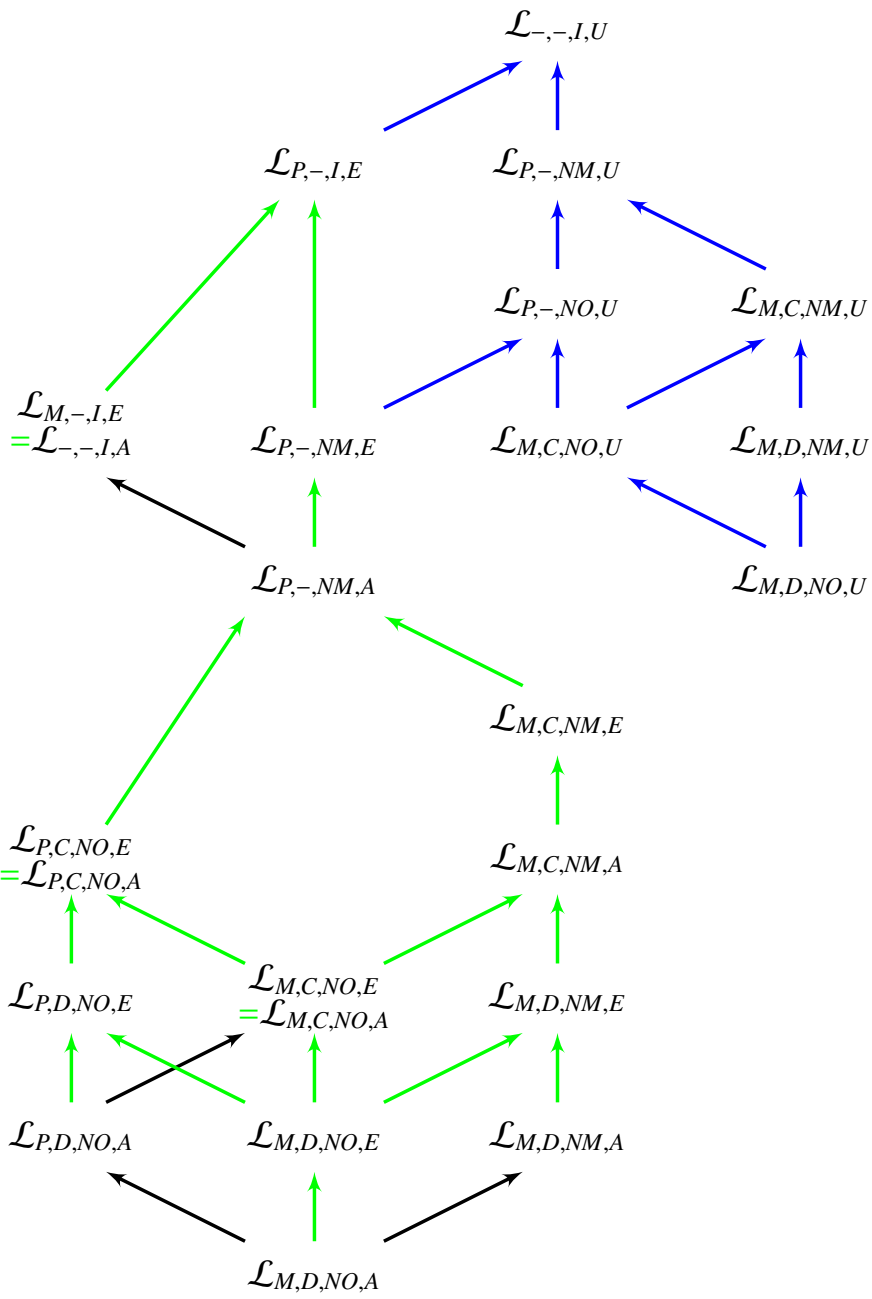
the choices of languages and encoding criteria, which corresponds to formalising Remark 1.

Proposition 4. If a language $\mathcal{L}_{1}$ is a sublanguage of $\mathcal{L}_{2}$ then there exists a valid encoding $\mathbb{I} \cdot \rrbracket$ from $\mathcal{L}_{1}$ into $\mathcal{L}_{2}$.

Finally, the existence of encodings $\mathbb{\|} \cdot \mathbb{1}_{1}$ from $\mathcal{L}_{1}$ into $\mathcal{L}_{2}$ and $\llbracket \cdot \mathbb{1}_{2}$ from $\mathcal{L}_{2}$ into $\mathcal{L}_{3}$ does not ensure that $\mathbb{[}\left[\mathbb{L} \cdot \mathbb{1}_{1} \mathbb{1}_{2}\right.$ is a valid encoding from $\mathcal{L}_{1}$ into $\mathcal{L}_{3}$ [17]. However, this does hold when the encodings use $\equiv$ rather than $\cong$ in the target language, as is the case for all encodings presented in this work. This allows later assumption of composition of encodings here, although this is not true for all valid encodings in general.

\section{Overview of Results}

A diagram illustrating the results can be seen in Figure 2. Arrows show increased expressive power and ='s show equivalence; black are from prior work, green from Section 5, and blue from Section 6. The lack of an arrow indicates no possible encoding in either direction (e.g. between $\mathcal{L}_{P,-, I, E}$ and $\mathcal{L}_{P,-, N M, U}$ ). Transitive relations are omitted (e.g. $\mathcal{L}_{P,-, N M, A}$ to $\left.\mathcal{L}_{P,-, N O, U}\right)$.

Fig. 2. Relations between all languages 


\section{Asymmetry and Exchange}

Exchange is a generalisation of asymmetric communication, i.e. $\mathcal{L}_{\alpha, \beta, \gamma, A}$ is trivially encoded by, $\mathcal{L}_{\alpha, \beta, \gamma, E}$ by Proposition 4 . The rest of this section details other relations between asymmetric and exchange languages.

\subsection{Exchange in Monadic Non-Intensional Languages}

This section considers the simpler languages and demonstrates the proof techniques to show that exchange cannot be easily encoded into asymmetry.

For the monadic non-intensional languages changing from asymmetric to exchange communication alone is almost always an increase in expressive power. The following result is presented to demonstrate the proof technique for the most complex. Simpler variations can be used to show that there exists no encoding of: $\mathcal{L}_{M, D, N O, E}$ into $\mathcal{L}_{M, D, N O, A}$, or $\mathcal{L}_{M, D, N M, E}$ into $\mathcal{L}_{M, D, N M, A}$.

Theorem 1. There exists no valid encoding of $\mathcal{L}_{M, C, N M, E}$ into $\mathcal{L}_{M, C, N M, A}$.

The exception to the general $\mathcal{L}_{M, \beta, \gamma, E}$ is more expressive than $\mathcal{L}_{M, \beta, \gamma, A}$ when $\gamma \neq I$ is $\mathcal{L}_{M, C, N O, E}$ into $\mathcal{L}_{M, C, N O, A}$. This is detailed in Section 5.2.

Within the monadic non-intensional exchange languages the usual diamond of relations exists where adding channel-based communication or name-matching are both increases in expressive power. The separation results between $\mathcal{L}_{M, D, N M, E}$ and $\mathcal{L}_{M, C, N O, E}$ is the most interesting result, as the rest can be proved via matching degree.

Theorem 2. The languages $\mathcal{L}_{M, D, N M, E}$ and $\mathcal{L}_{M, C, N O, E}$ are unrelated.

\subsection{Encoding Exchange into Asymmetry}

This section considers where exchange languages can be encoded by asymmetric languages. Note that this does not ensure atomicity that motivates some languages [10].

An exchange language $\mathcal{L}_{1}$ can be encoded into an asymmetric language $\mathcal{L}_{2}$ if the matching degree of $\mathcal{L}_{1}$ is bounded, and: $\mathcal{L}_{1}$ and $\mathcal{L}_{2}$ are both channel-based no-matching languages; or $\mathcal{L}_{2}$ has a greater matching degree and is polyadic or channel-based.

In the first case, the key idea is to represent the channel name by a pair of names to indicate whether the input is on the action or co-action. Consider the following translation from $\mathcal{L}_{M, C, N O, E}$ into $\mathcal{L}_{M, C, N O, A}$ :

$$
\begin{array}{r}
\llbracket(v n) P \rrbracket \stackrel{\text { def }}{=}\left(v n_{1}\right)\left(v n_{2}\right) \llbracket P \rrbracket \\
\llbracket n(\lambda x) \cdot P \rrbracket \stackrel{\text { def }}{=} n_{1}(\lambda \mathrm{rn}) \cdot \operatorname{rn}\left(\lambda x_{1}\right) \cdot x\left(\lambda x_{2}\right) \cdot \llbracket P \rrbracket \\
\llbracket \bar{n}\langle\lambda x\rangle \cdot P \rrbracket \stackrel{\text { def }}{=} n_{2}(\lambda \mathrm{rn}) \cdot \operatorname{rn}\left(\lambda x_{1}\right) \cdot x\left(\lambda x_{2}\right) \cdot \llbracket P \rrbracket \\
\llbracket n(a) \cdot P \rrbracket \stackrel{\text { deff }}{=}(v \mathrm{rn}) \overline{n_{2}}\langle\mathrm{rn}\rangle \cdot \overline{\mathrm{rn}}\left\langle a_{1}\right\rangle \cdot \overline{\mathrm{rn}}\left\langle a_{2}\right\rangle \cdot \llbracket P \rrbracket \\
\llbracket \bar{n}\langle a\rangle \cdot P \rrbracket \stackrel{\text { def }}{=}(v \mathrm{rn}) \overline{n_{1}}\langle\mathrm{rn}\rangle \cdot \overline{\mathrm{rn}}\left\langle a_{1}\right\rangle \cdot \overline{\mathrm{rn}}\left\langle a_{2}\right\rangle \cdot \llbracket P \rrbracket \\
\llbracket \text { if } s=t \text { then } P \text { else } Q \rrbracket \stackrel{\text { def }}{=} \text { if } s_{1}=t_{1} \text { then } \llbracket P \rrbracket \text { else } \mathbb{\|} Q \rrbracket .
\end{array}
$$

Here the names $n_{1}$ and $n_{2}$ represent two parts of the name $n$, and $r n$ is a reserved name, these are all introduced by the renaming policy $\varphi_{\mathbb{I} \Perp}[18,11]$. 
Theorem 3. The encoding from $\mathcal{L}_{M, C, N O, E}$ into $\mathcal{L}_{M, C, N O, A}$ is valid.

The above encoding illustrates how channel-based communication is sufficient when no name-matching or intensionality is included in the source language.

In the second case, the key idea is that a single name is sufficient to represent the shape of the encoded action or co-action, and so can ensure correct encoded interactions. Observe that in every case the reverse encoding is proved impossible easily via the matching degree and Proposition 2. The clearest illustration of this when the source language is monadic is the following encoding from $\mathcal{L}_{M, D, N O, E}$ into $\mathcal{L}_{M, C, N O, A}$. Consider the translation $\llbracket \cdot \rrbracket$ that is homeomorphic on all forms except for the action and co-action, and exploits two reserved names ia and ic that are translated as follows:

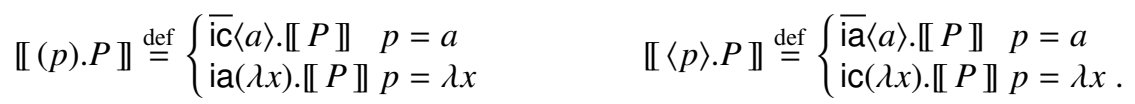

The channel name indicates the origin of the input, ia for action, and ic for co-action.

Theorem 4. The encoding from $\mathcal{L}_{M, D, N O, E}$ into $\mathcal{L}_{M, C, N O, A}$ is valid.

The existence of an encoding from $\mathcal{L}_{M, D, N M, E}$ into $\mathcal{L}_{M, C, N M, A}$ is achieved in the same manner by extending the initial translation to consider name matches $\ulcorner a\urcorner$ to also be inputs, e.g. i.e. $\llbracket(\ulcorner a\urcorner) . P \rrbracket \stackrel{\text { def }}{=} \operatorname{ia}(\ulcorner a\urcorner)$. $\llbracket P \rrbracket$.

The existence of valid encodings from $\mathcal{L}_{M, C, N M, E}$ into $\mathcal{L}_{P,-, N M, A}$ can be shown with a similar technique, instead of the reserved names being used as a channel they are simply added as another part of the polyadic input or output (with name-matching on the input). For example, $\llbracket n(a) . P \rrbracket \stackrel{\text { def }}{=} \overline{\mathrm{ic}}\langle n, a\rangle$ and $\llbracket n(\lambda x) . P \rrbracket \stackrel{\text { def }}{=} \mathrm{ia}(\ulcorner n\urcorner, \lambda x)$.

A similar but more complex technique can be used to encode polyadic no-matching exchange languages into asymmetric languages. This is illustrated by the following encoding from $\mathcal{L}_{P, D, N O, E}$ into $\mathcal{L}_{P, C, N O, A}$. The encoding exploits a binary representation of the structure of an action or co-action. To this end define the binary representation function Bin(.) that converts a sequence of names and binding names into a bit-string and also the complement (or bitwise not) Not(.) of bit-strings (where ';' is concatenation):

$$
\begin{array}{lll}
\operatorname{BiN}(a)=\mathbb{0} & \operatorname{Bin}(\lambda x)=1 & \operatorname{Bin}(n, \widetilde{n})=\operatorname{BIN}(n) ; \operatorname{Bin}(\widetilde{n}) \\
\operatorname{Not}(\mathbb{Q})=1 & \operatorname{Not}(1)=\mathbb{0} & \operatorname{Not}(\mathbf{X}, \widetilde{\mathrm{X}})=\operatorname{Not}(\mathbf{X}) ; \operatorname{Not}(\widetilde{\mathrm{X}}) .
\end{array}
$$

Given a sequence of binding names and names $\widetilde{p}$, the sequences of the binding names $\operatorname{BN}(\widetilde{p})$, and names $\mathrm{NM}_{\mathrm{M}}(\widetilde{p})$ are defined by:

$$
\begin{array}{ll}
\operatorname{BN}(\lambda x, \widetilde{p})=\lambda x, \operatorname{BN}(\widetilde{p}) & \operatorname{BN}(a, \widetilde{p})=\operatorname{BN}(\widetilde{p}) \\
\operatorname{NM}(\lambda x, \widetilde{p})=\operatorname{NM}(\widetilde{p}) & \operatorname{NM}(a, \widetilde{p})=a, \operatorname{NM}(\widetilde{p}) .
\end{array}
$$

Now consider the translation $\mathbb{[} \cdot \rrbracket$ that is homeomorphic on all forms except the action and co-action (and exploits a reserved name $\mathrm{rn}$ ) that are translated as follows:

$$
\begin{aligned}
& \llbracket(\widetilde{p}) \cdot P \rrbracket \stackrel{\text { def }}{=} a(\lambda \mathrm{rn}, \operatorname{BN}(\widetilde{p})) \cdot \overline{\operatorname{rn}}\langle\operatorname{Nm}(\widetilde{p})\rangle \cdot \llbracket P \rrbracket \quad a=\operatorname{BIN}(\widetilde{p}) \\
& \llbracket\langle\widetilde{p}\rangle \cdot P \rrbracket \stackrel{\text { def }}{=}(v \mathrm{rn}) \bar{a}\langle\mathrm{rn}, \operatorname{NM}(\widetilde{p})\rangle \cdot \operatorname{rn}(\operatorname{BN}(\widetilde{p})) \cdot \llbracket P \rrbracket a=\operatorname{Not}(\operatorname{BIN}(\widetilde{p})) .
\end{aligned}
$$


The idea is that the translated action and co-action match on the channel name that is the bit-string representation of their order of binding names and names. If they match the input performs all the action's bindings as well as an additional name (bound to) $\mathrm{rn}$. The rôles are then reversed to complete the interaction.

Theorem 5. The encoding from $\mathcal{L}_{P, D, N O, E}$ into $\mathcal{L}_{P, C, N O, A}$ is valid.

The encoding from $\mathcal{L}_{P, C, N O, E}$ into $\mathcal{L}_{P, C, N O, A}$ exploits elements of the technique above. Define the function $\operatorname{VAL}(\cdot)$ that gives the numeric value of a binary string, e.g. $\operatorname{VaL}(101)=$ 5 and $\operatorname{VAL}(1010)=10$. Now the encoding from $\mathcal{L}_{P, C, N O, E}$ into $\mathcal{L}_{P, C, N O, A}$ can be constructed as follows exploiting a reserved name $\mathrm{rn}$ as usual:

$$
\begin{gathered}
\llbracket n(\widetilde{p}) . P \rrbracket \stackrel{\text { def }}{=} n\left(\lambda \mathrm{rn}, \operatorname{BN}(\widetilde{p}), \lambda z, \ldots, \lambda z_{i}\right) \cdot \overline{\mathrm{n}}\langle\operatorname{NM}(\widetilde{p})\rangle \cdot \llbracket P \rrbracket \\
\text { where } i=\operatorname{VAL}(1 ; \operatorname{BIN}(\widetilde{p}))-|\operatorname{BN}(\widetilde{p})| \\
\llbracket \bar{n}\langle\widetilde{p}\rangle . P \rrbracket \stackrel{\text { def }}{=}(v \mathrm{rn})\left(v z_{1}\right) \ldots\left(v z_{i}\right) \bar{n}\left\langle\operatorname{rn}, \operatorname{NM}(\widetilde{p}), z_{1}, \ldots, z_{i}\right\rangle \cdot \operatorname{rn}(\operatorname{BN}(\widetilde{p})) \cdot \llbracket P \rrbracket \\
\text { where } i=\operatorname{VAL}(1 ; \operatorname{Not}(\operatorname{Bin}(\widetilde{p})))-|\operatorname{NM}(\widetilde{p})|
\end{gathered}
$$

and translating all other processes homomorphically. Also $\widetilde{z}$ do not intersect one another, or any of the names in $n$ and $\widetilde{p}$ and $f n(P)$.

The key idea is to map the binary representation of the structure of the action or co-action to the arity of the encoded action or co-action. To prevent conflicts between encodings, for example $n(a, \lambda x)$ and $n(\lambda x)$, the binary representation is pre-pended with 1 . Thus, the arity of the action or co-action ensures correct interaction if the structure is correct, and the channel name is matched as usual.

Theorem 6. The encoding from $\mathcal{L}_{P, C, N O, E}$ into $\mathcal{L}_{P, C, N O, A}$ is valid.

Building on Theorem 5 and the equivalence between the languages $\mathcal{L}_{P,-, N M, A}[16]$ conclude that $\mathcal{L}_{P,-N M, A}$ are able to encode all the: monadic non-intensional exchange languages; and the polyadic no-matching exchange languages.

\subsection{Other Relations With Bounded Matching Degree}

This section considers other relations between languages equally or less expressive than $\mathcal{L}_{P,-, N M, A}$, i.e. all the languages that can be encoded in $\mathcal{L}_{P,-, N M, A}$.

Within exchange languages, clearly $\mathcal{L}_{M, \beta, N O, E}$ is a sub-language of $\mathcal{L}_{P, \beta, N O, E}$ for any $\beta$ and so can be validly encoded by Proposition 4 . The following proves the separation results required to indicate an increase in expressiveness.

Theorem 7. There exists no valid encoding of $\mathcal{L}_{P, D, N O, E}$ into $\mathcal{L}_{M, D, N O, E}$.

Observe that this result can be used to show there exists no valid encoding of $\mathcal{L}_{P, C, N O, E}$ into $\mathcal{L}_{M, C, N O, E}$ by having all communication along a single channel name and preventing modification of this name by the encoding.

Regarding asymmetric languages, $\mathcal{L}_{P, D, N O, E}$ can validly encode $\mathcal{L}_{P, D, N O, A}$ by Proposition 4 . The following proves an increase in expressiveness.

Theorem 8. There exists no valid encoding of $\mathcal{L}_{P, D, N O, E}$ into $\mathcal{L}_{P, D, N O, A}$. 


\subsection{Equivalent Languages with Unbounded Matching Degree}

Once the matching degree is unbounded several languages become equivalent in expressiveness, this section formalises these results.

The intensional asymmetric languages all have equivalent expressiveness (by Theorem 6.5 of [12]) and to the monadic exchange languages. Consider the languages $\mathcal{L}_{M, D, I, E}$ and $\mathcal{L}_{M, C, I, E}$, there is a trivial valid encoding of $\mathcal{L}_{M, D, I, E}$ into $\mathcal{L}_{M, C, I, E}$ by Proposition 4 . The following shows equivalence via the reverse encoding from $\mathcal{L}_{M, C, I, E}$ into $\mathcal{L}_{M, D, I, E}$. Take the encoding $\mathbb{\|} \cdot \rrbracket$ that is the homeomorphic on all processes except the action and co-action that are encoded as follows (exploiting reserved names as usual):

$$
\left.\begin{array}{l}
\llbracket p(q) . P \rrbracket \stackrel{\text { def }}{=}\langle\text { ic } \bullet p \bullet q\rangle . \llbracket P \rrbracket \\
\llbracket \bar{p}\langle q\rangle . P \rrbracket \stackrel{\text { def }}{=}\langle\text { ia } \bullet p \bullet q\rangle . \llbracket P \rrbracket
\end{array}\right\} q \text { is a term }
$$

The translation compounds the channel pattern $p$ with the term or intensional pattern $q$, converting to maintain being either a term or intensional pattern.

Theorem 9. The encoding from $\mathcal{L}_{M, C, I, E}$ into $\mathcal{L}_{M, D, I, E}$ is valid.

Now to complete the equivalences. Since all the languages $\mathcal{L}_{-,-, I, A}$ are equally expressive and since the languages $\mathcal{L}_{M,-, I, E}$ are equally expressive by Theorem 9 it suffices to consider examples from either group. The encodings from $\mathcal{L}_{-,-, I, A}$ into $\mathcal{L}_{M,-, I, E}$ follow by $\mathcal{L}_{M, D, I, A}$ being a sub-language of $\mathcal{L}_{M, D, I, E}$. In the other direction, there exists a valid encoding from $\mathcal{L}_{M, D, I, E}$ into $\mathcal{L}_{M, C, I, A}$, by a straightforward adaption of Theorem 4 .

Considering polyadic non-intensional languages, $\mathcal{L}_{P, D, N M, E}$ can be encoded into $\mathcal{L}_{P, C, N M, E}$ by Proposition 4 . For the converse, the standard approach $[16,12]$ yields a valid encoding; one that is homeomorphic on all forms except the action $\llbracket a(\widetilde{p}) . P \rrbracket \stackrel{\text { def }}{=}$ $(\ulcorner a, \widetilde{p}) . \llbracket P \rrbracket$ and co-action $\llbracket \bar{a}\langle\vec{p}\rangle . P \rrbracket \stackrel{\text { def }}{=}\langle a, \widetilde{p}\rangle . \llbracket P \rrbracket$. Indeed this approach can be used for the polyadic intensional languages, showing the existence of a valid encoding from $\mathcal{L}_{P, C, I, E}$ into $\mathcal{L}_{P, D, I, E}$. Equivalence is completed by showing a valid encoding of $\mathcal{L}_{P, D, I, E}$ into $\mathcal{L}_{P, C, I, E}$ by Proposition 4.

\subsection{Concluding Relations}

This section concludes the relations between asymmetric and exchange languages by formalising those between languages with unbounded matching degree. In general this is showing separation results between different language groups.

Polyadic intensional exchange languages are more expressive than any other exchange or asymmetric languages. By the encodings in Section 5.4 in all languages considered here being dataspace-based or channel-based is immaterial to expressive power. The languages $\mathcal{L}_{P,-, N M, E}$ are sub-languages of $\mathcal{L}_{P,-, I, E}$ and so their expressiveness is included naturally, the reverse is from the following result.

Theorem 10. There exists no valid encoding of $\mathcal{L}_{P,-, I, E}$ into $\mathcal{L}_{P,-, N M, E}$. 
Comparing within other intensional exchange languages, $\mathcal{L}_{P,-, I, E}$ can encode $\mathcal{L}_{M,-, I, E}$ by Proposition 4. The reverse separation result uses the technique of Theorem 11.

That the languages $\mathcal{L}_{M,-, I, E}$ are unrelated to $\mathcal{L}_{P,-, N M, E}$ follows from the separation results that show no valid encodings from $\mathcal{L}_{M,-, I, E}$ into $\mathcal{L}_{P,-, N M, E}$, and $\mathcal{L}_{P,-, N M, E}$ into $\mathcal{L}_{M,-, I, E}$ (proved using the techniques of Theorems $10 \& 7$, respectively). Note the groups of languages can be treated equivalently due to the encodings of Section 5.4.

Lastly, the languages $\mathcal{L}_{P,-, N M, A}$ can be encoded $\mathcal{L}_{P,-, N M, E}$ via Proposition 4 . The reverse is prevented by the following result.

Theorem 11. There exists no valid encoding from $\mathcal{L}_{P,-, N M, E}$ into $\mathcal{L}_{P,-, N M, A}$.

\section{Unification}

This section considers the expressiveness of unification languages, and their relations to asymmetric and exchange languages.

\subsection{Unification Cannot be Simulated}

The following result shows that no unification language can be encoded into an asymmetric or exchange language. Key is a self recognising process, defined to be $S=(a) \cdot \sqrt{ }$ for the dataspace-based languages and $S=a(a) \cdot \sqrt{ }$ for the channel-based languages, that has the behaviour $S \mid S \longmapsto \Downarrow$ but $S \mapsto$ and $S \Downarrow$. This can be exploited since no non-unification process can reduce in parallel with itself unless it reduces alone. The self recognising process can be used to yield the following result via Proposition 3.

Theorem 12. There exists no valid encoding of a unification language $\mathcal{L}_{-,-,-, U}$ into any non-unification language $\mathcal{L}_{-,-,-, \delta} \delta \neq U$.

The above result can be used to prove a separation result from any unification language to a non-unification language, these results are omitted from the rest of the paper.

\subsection{On Monadic Non-Intensional Unification Languages}

All the languages $\mathcal{L}_{M,-, \gamma, E}$ where $\gamma \neq I$ are unrelated to any non-unification language. Similar to the languages $\mathcal{L}_{M,-, \gamma, A}$, these 4 form a diamond where expressiveness is increased by adding channel-based communication or pattern-matching.

The shift to unification leads to $\mathcal{L}_{M, D, N O, U}$ being unrelated to any other language $\mathcal{L}_{M, D, N O,-}$. The following result illustrates how to achieve such separation results and can be applied to other monadic non-intensional languages also.

Theorem 13. There exists no valid encoding from $\mathcal{L}_{M, D, N O, \delta}$ where $\delta \neq U$ into $\mathcal{L}_{M, D, N O, U}$.

The relations between the monadic non-intensional unification languages are as usual, although the usual proof techniques do not always hold. In particular, no-matching unification languages still have non-zero matching degree, so separation results that rely on matching degree alone no longer hold. $\mathcal{L}_{M, D, N O, U}$ can be validly encoded by $\mathcal{L}_{M, D, N M, U}$ via Proposition 4 . The following proves the separation result. 
Theorem 14. There exists no valid encoding of $\mathcal{L}_{M, D, N M, U}$ into $\mathcal{L}_{M, D, N O, U}$.

The above technique can be applied to prove that there exist no encodings from $\mathcal{L}_{M, C, N M, U}$ into $\mathcal{L}_{M, C, N O, U}$, or from $\mathcal{L}_{M, D, N M, U}$ into $\mathcal{L}_{M, C, N O, U}$ The rest of the separation results to prove that the relations are the same as in the asymmetric setting exploit the matching degree of the languages involved.

\subsection{Equally Expressive Unification Languages}

Once the matching degree is unbounded there is no difference in expressiveness between dataspace-based and channel-based communication for unification languages. Further, all the intensional unification languages have equal expressive power.

For the polyadic languages it is straightforward to represent channel-based communication by shifting the channel to the first position of a dataspace-based encoding. For both encodings from $\mathcal{L}_{P, C, N O, U}$ into $\mathcal{L}_{P, D, N O, U}$ and $\mathcal{L}_{P, C, N M, U}$ into $\mathcal{L}_{P, D, N M, U}$ are achieved by $\llbracket a(\widetilde{p}) . P \rrbracket \stackrel{\text { def }}{=}(a, \widetilde{p})$. $\llbracket P \rrbracket$. The converse results are by Proposition 4 .

This may at first appear unexpected since in the asymmetric and exchange languages $\mathcal{L}_{P, C, N O, \delta}(\delta \neq U)$ have matching degree 1 while $\mathcal{L}_{P, D, N O, \delta}(\delta \neq U)$ have matching degree 0 . However, this does not hold for unification languages as due to the poly-unify rule their matching degree directly relates to their arity.

All the intensional unification languages are equally expressive. Clearly the languages $\mathcal{L}_{M,-, I, U}$ and $\mathcal{L}_{-, D, I, U}$ can be trivially validly encoded into the languages $\mathcal{L}_{P,-, I, U}$ and $\mathcal{L}_{-, C, I, U}$, respectively, by sub-language inclusion. An encoding from $\mathcal{L}_{P,-, I, U}$ into $\mathcal{L}_{M,-, I, U}$ can be easily achieved in the same manner as Theorem 5.4 of [12] by encoding the polyadic structure into a monadic intensional pattern. The key idea is that a sequence of patterns $\widetilde{p}=p_{1}, \ldots, p_{i}$ is encoded as a single pattern $\left(\mathrm{rn} \bullet p_{1}\right) \bullet \ldots \bullet p_{i}$ where $\mathrm{rn}$ is a reserved name. For showing an encoding from $\mathcal{L}_{-, C, I, U}$ into $\mathcal{L}_{-, D, I, U}$ the same technique as used in Theorem 9 can be used.

\subsection{Encodings into Polyadic Non-Intensional Languages}

This section considers encodings into polyadic non-intensional unification languages. Despite being nominally no-matching it is still possible to encode polyadic namematching into the languages $\mathcal{L}_{P,-, N O, U}$. Beyond this the usual increases in expressiveness hold for shifting from monadic to polyadic, and from no-matching to name-matching. The rest of this section details these relations.

Unification communication exploits pattern unification that allows equivalence of patterns. The key difference is that a single name can unify with itself unlike in the poly-match rule where $\operatorname{Match}(a, a)$ is undefined. This breaks the directionality assumed in asymmetric and exchange primitives, and so invalidates many prior results.

The directionality of asymmetric or exchange languages can be maintained by an encoding when the target language is either polyadic or intensional. Define the unprotect function $g$ that replaces all instances of $\ulcorner a\urcorner$ with $a$ in a pattern. Consider the encoding $\llbracket \cdot \rrbracket$ from $\mathcal{L}_{P, D, N M, E}$ to $\mathcal{L}_{P, C, N O, U}$ that exploits the functions BIN and Not of 
Section 5.2 and is homeomorphic on all forms except as defined below:

$$
\begin{array}{ll}
\llbracket(\widetilde{p}) \cdot P \rrbracket \stackrel{\text { def }}{=} a(\lambda \mathrm{rn}, \widetilde{g(p)}) \cdot \llbracket P \rrbracket & a=\operatorname{Bin}(\widetilde{p}) \\
\llbracket\langle\widetilde{p}\rangle \cdot P \rrbracket \stackrel{\text { def }}{=}(v \mathrm{rn}) a(\mathrm{rn}, \widetilde{g(p)}) . \llbracket P \rrbracket & a=\operatorname{Not}(\operatorname{Bin}(\widetilde{p}))
\end{array}
$$

The binary encoding is used to ensure that inputs and outputs are properly aligned since otherwise two outputs may unify. The additional reserved name is to distinguish actions from co-actions in the translation. The unprotect function $g$ converts name-matches into names since the former aren't defined in a no-matching language.

Theorem 15. The encoding from $\mathcal{L}_{P, D, N M, E}$ into $\mathcal{L}_{P, C, N O, U}$ is valid.

Observe that since $\mathcal{L}_{P, D, N M, E}$ generalises the languages $\mathcal{L}_{-,-, \gamma, \delta}$ where $\gamma \leq N M$ and $\delta \leq E$ this proof applies to all such languages.

Regarding other unification languages, clearly $\mathcal{L}_{P,-, N O, U}$ can be validly encoded by $\mathcal{L}_{P,-, N M, U}$, with shifts between dataspace-based and channel-based communication handled by the encodings of Section 6.3 and Proposition 4. The separation result required to indicate an increase in expressiveness from $\mathcal{L}_{P,-, N O, U}$ to $\mathcal{L}_{P,-, N M, U}$ can be proved using the same technique as Theorem 14 . The relations between polyadic and monadic languages are as expected. $\mathcal{L}_{M, C, N O, U}$ can be encoded by $\mathcal{L}_{P, C, N O, U}$ via Proposition 4 , and thus also $\mathcal{L}_{P, D, N O, U}$ by encoding from Section 6.3. The separation result that $\mathcal{L}_{M, C, N O, U}$ cannot encode $\mathcal{L}_{P,-, N O, U}$ is by Proposition 2 . Similar results hold for the name-matching languages also. $\mathcal{L}_{M, C, N M, U}$ can be encoded by $\mathcal{L}_{P, C, N M, U}$ by Proposition 4 (and thus also $\mathcal{L}_{P, D, N M, U}$ by encoding from Section 6.3). The reverse separation that there exists no valid encoding of $\mathcal{L}_{P,-, N M, U}$ into $\mathcal{L}_{M, C, N M, U}$ is proven via Proposition 2.

\subsection{Intensional Unification Languages}

Since all the intensional unification languages are equivalent by exploiting encodings from Section 6.3, it remains to show their other relations.

The intensional unification languages can also encode directionality in a similar manner to Section 6.4 (Theorem 15). Consider the encoding $\llbracket \cdot \rrbracket$ from $\mathcal{L}_{P, D, I, E}$ to $\mathcal{L}_{M, C, I, U}$ that exploits the numerical encoding function BIN and Not of Sections $5.2 \& 6.4$ and is the homeomorphic on all forms except the action and co-action:

$$
\begin{array}{lll}
\mathbb{}\left(p_{1}, \ldots, p_{i}\right) . P \rrbracket & \stackrel{\text { def }}{=} a\left(\lambda \mathrm{rn} \bullet\left(p_{1} \bullet \ldots \bullet p_{i}\right)\right) . \llbracket P \rrbracket & a=\operatorname{BIN}(\widetilde{p}) \\
\llbracket\left\langle p_{1}, \ldots, p_{i}\right\rangle . P \rrbracket \stackrel{\text { def }}{=} a\left(\mathrm{rn} \bullet\left(p_{1} \bullet \ldots \bullet p_{i}\right)\right) . \llbracket P \rrbracket & a=\operatorname{Not}(\operatorname{Bin}(\widetilde{p}))
\end{array}
$$

where $\mathrm{rn}$ is a reserved name as usual. The translations of actions and co-actions are as before except that compounding is used in place of polyadic sequencing.

Theorem 16. The encoding from $\mathcal{L}_{P, D, I, E}$ into $\mathcal{L}_{M, C, I, U}$ is valid.

$\mathcal{L}_{-,-, I, U}$ are more expressive than $\mathcal{L}_{P,-, I, E}$ since there exists an encoding from $\mathcal{L}_{P, D, I, E}$ into $\mathcal{L}_{M, C, I, U}$ by Theorem 16, conclude via encodings of Sections $5.4 \& 6.3$.

Finally, within unification languages intensionality remains more expressive than non-intensionality. By encodings in Section 6.3 all languages considered here being channel-based or dataspace-based is immaterial. The languages $\mathcal{L}_{P,-, N M, U}$ can be encoded by $\mathcal{L}_{P,-, I, U}$ by Proposition 4 , the final separation of $\mathcal{L}_{P,-, I, U}$ into $\mathcal{L}_{P,-, N M, U}$ can be proved using the techniques of Theorem 10 and completes the results. 


\section{Conclusions and Discussion}

Symmetric communication primitives provide new and interesting perspectives on how languages and communication can occur. Considering exchange provides interesting insight into how much trading systems and atomic exchange actions can be captured within asymmetric languages by encoding. The ability to encode polyadic exchange without name matching into asymmetric languages indicates that it is the addition of (unbounded, or multiple) name-matching with exchange that really extends expressiveness. While exchange generally increases expressiveness over asymmetric languages, it is pattern-matching that provides the strongest expressiveness alone. The unification languages cannot be encoded into even exchange languages, the self recognising technique was used for CPC before [14] but is here generalised. The flexibility of unification allows for names to be matched even in a language that nominally does not have name-matching. This yields some interesting results where non-matching languages can encode name-matching languages by exploiting unification. However, name-matching still provides increased expressiveness within unification languages, and intensionality is the sole factor in determining the most expressive language.

Choices of Primitives The choices of primitives here is to align with prior work and results $[16,18,12]$. However, there are other choices that would impact some results.

The patterns are chosen here to match those of CPC and it turns out that the (symmetric) patterns here are sufficient to represent most other approaches, such as Spi Calculus [14] and Psi Calculi terms [12]. More generally the core approach of compounding proves sufficient to represent many complex data structures and even (in practice) type information. This has been discussed and formalised in different settings [19, 20, $10]$ and in many works related to pattern calculus, $S F$-logic, and CPC.

For the process forms the most obvious alternative would be to consider a choice operator: $\alpha_{1} . P+\alpha_{2} \cdot Q$ for some choice of $\alpha_{i}$. Again the decision not to include this is to match with prior results $[16,18,12]$. The addition of such a choice operator could invalidate some findings, in particular Theorem 12. This provides illustration of which results would need to be reexamined with such a change, although it does not (a priori) indicate that the overall relative expressiveness would change. For example, previous simple results for the inability to encode $\operatorname{CPC}\left(\mathcal{L}_{M, D, I, F}\right)$ into $\pi$-calculus $\left(\mathcal{L}_{M, C, N O, A}\right)$ have used this approach [14], however alternative proofs also exist such as Proposition 2 and Theorems $11, \& 14$ and in prior works $[16,12]$. This lends weight to the rigour here that provides alternative approaches, and identifies which results rely on which primitives.

In this context there are many other possible choices of primitives for both the patterns and the processes. However, those here are sufficient to understand the core dynamics between the interaction features of languages. Also by using a common approach that is transitive for the encodings here, often more distant relations can be proved without relying on particular choices of primitives or proof techniques.

Related Work This section provides a brief account of related works most close to the decisions and results here, since to cover all related works would take an entire paper.

There are already existing specific results for some symmetric process calculi that agree with the results here. CPC $\left(\mathcal{L}_{M, D, I, U}\right)$ can homomorphically encode: $\pi$-calculus 
( $\left.\mathcal{L}_{M, C, N O, A}\right)$, Linda $\left(\mathcal{L}_{P, D, N M, A}\right)$, and Spi Calculus (perhaps $\left.\mathcal{L}_{M, C, I, A}\right)$ while none of them can encode CPC $[14,12]$. Similarly fusion calculus can encode $\pi$-calculi, although not the other way around [28]. Impossibility of encoding results for CPC and fusion calculus into many calculi can be derived from the results here. Fusion calculus and Psi calculi are unrelated to CPC in that neither can encode CPC, and CPC cannot encode either of them $[10,14]$. However, these results rely upon the global effect of fusions in fusion calculus, and the inclusing of logic in Psi Calculi.

There are also related works on concurrent constraint languages (CCL) [6, 7]. The encoding criteria of [7] are similar to those here, although the authors assume that parallel composition must be encoded homomorphically. This holds for all the encodings presented in this paper, but not for the separation results (making them stronger, although the proofs a little harder). However, CCLs have a different communication paradigm, with interaction between a single process and a common store of constraints, which is quite different to the focus of this paper. However, such non-binary approaches to communications have been considered $[8,15]$. Further, the expressiveness of CCLs depends to some degree on the logic, which is again not considered as part of communication paradigms here (although since CCLs and Psi Calculi exploit logics, this may be an interesting path of future research). In [6] there is also unification of terms, however their approach is different in that unifying $s$ and $t$ by $\sigma$ is achieved when $\sigma s=\sigma t$. It is possible to restructure the unification rule here to use a single substitution (although this is overly complex and requires reasoning over processes not just patterns), but the unification would still differ since there is no distinction for name-matches $\ulcorner a$ in [6].

\section{References}

1. J. Bengtson, M. Johansson, J. Parrow, and B. Victor. Psi-calculi: a framework for mobile processes with nominal data and logic. Logical Methods in Computer Science, 7(1), 2011.

2. J. Bengtson and J. Parrow. Formalising the pi-calculus using nominal logic. Logical Methods in Computer Science, 5(2), 2009.

3. G. Boudol. Notes on algebraic calculi of processes. In Logics and Models of Concurrent Systems, pages 261-303. Springer-Verlag, New York, NY, USA, 1985.

4. N. Busi, R. Gorrieri, and G. Zavattaro. On the expressiveness of LindA coordination primitives. Information and Computation, 156(1-2):90-121, 2000.

5. M. Carbone and S. Maffeis. On the expressive power of polyadic synchronisation in $\pi$ calculus. Nordic Journal of Computing, 10(2):70-98, May 2003.

6. F. S. de Boer and C. Palamidessi. Concurrent logic programming: Asynchronism and language comparison. In Proceedings of the 1990 North American Conference on Logic Programming, pages 175-194, Cambridge, MA, USA, 1990. MIT Press.

7. F. S. Deboer and C. Palamidessi. Embedding as a tool for language comparison. Information and computation, 108(1):128-157, 1994.

8. C. Fournet and G. Gonthier. The reflexive cham and the join-calculus. In Proceedings of the 23rd ACM Symposium on Principles of Programming Languages, pages 372-385. ACM Press, 1996.

9. D. Gelernter. Generative communication in Linda. ACM Transactions on Programming Languages and Systems, 7(1):80-112, 1985.

10. T. Given-Wilson. Concurrent Pattern Unification. PhD thesis, University of Technology, Sydney, Australia, 2012. 
11. T. Given-Wilson. An intensional concurrent faithful encoding of Turing machines. In Proceedings ICE 2014, Berlin, Germany, 6th June 2014., pages 21-37, 2014.

12. T. Given-Wilson. On the Expressiveness of Intensional Communication. In Proceedings of EXPRESS/SOS, Rome, Italie, Sept. 2014.

13. T. Given-Wilson and D. Gorla. Pattern matching and bisimulation. In Coordination Models and Languages, volume 7890 of LNCS, pages 60-74. Springer Berlin Heidelberg, 2013.

14. T. Given-Wilson, D. Gorla, and B. Jay. A Concurrent Pattern Calculus. Logical Methods in Computer Science, 10(3), 2014.

15. T. Given-Wilson and A. Legay. On the Expressiveness of Joining. In ICE 2015, Grenoble, France, June 2015.

16. D. Gorla. Comparing communication primitives via their relative expressive power. Information and Computation, 206(8):931-952, 2008.

17. D. Gorla. A taxonomy of process calculi for distribution and mobility. Distributed Computing, 23(4):273-299, 2010.

18. D. Gorla. Towards a unified approach to encodability and separation results for process calculi. Information and Computation, 208(9):1031-1053, 2010.

19. B. Jay. Pattern Calculus: Computing with Functions and Data Structures. Springer, 2009.

20. B. Jay and T. Given-Wilson. A combinatory account of internal structure. Journal of Symbolic Logic, 76(3):807-826, 2011.

21. I. Lanese, J. Prez, D. Sangiorgi, and A. Schmitt. On the expressiveness of polyadic and synchronous communication in higher-order process calculi. In Automata, Languages and Programming, volume 6199 of LNCS, pages 442-453. Springer Berlin Heidelberg, 2010.

22. I. Lanese, C. Vaz, and C. Ferreira. On the expressive power of primitives for compensation handling. In Proceedings of the 19th European Conference on Programming Languages and Systems, ESOP'10, pages 366-386, Berlin, Heidelberg, 2010. Springer-Verlag.

23. R. Milner. The polyadic $\pi$-calculus: A tutorial. In Logic and Algebra of Specification, volume 94 of Series F. NATO ASI, Springer, 1993.

24. R. Milner, J. Parrow, and D. Walker. A calculus of mobile processes, I \& II. Information and Computation, 100(1):1-77, Sept. 1992.

25. L. Nielsen, N. Yoshida, and K. Honda. Multiparty symmetric sum types. In Proceedings of EXPRESS, pages 121-135, 2010.

26. C. Palamidessi. Comparing the expressive power of the synchronous and asynchronous picalculi. Mathematical. Structures in Comp. Sci., 13(5):685-719, Oct. 2003.

27. J. Parrow. Expressiveness of process algebras. Electronic Notes in Theoretical Computer Science, 209:173-186, Apr. 2008.

28. J. Parrow and B. Victor. The fusion calculus: expressiveness and symmetry in mobile processes. In Proceedings of 13th Annual IEEE Symposium on Logic in Computer Science, pages 176-185, Jun 1998.

29. K. Peters. Translational expressiveness: comparing process calculi using encodings. $\mathrm{PhD}$ thesis, Technische Universität Berlin, Fakultät IV, Germany, 2012.

30. E. Shapiro. Separating concurrent languages with categories of language embeddings. In Proceedings of the Twenty-third Annual ACM Symposium on Theory of Computing, STOC '91, pages 198-208, New York, NY, USA, 1991. ACM.

31. R. J. van Glabbeek. Musings on encodings and expressiveness. In Proceedings of EXPRESS/SOS, volume 89 of EPTCS, pages 81-98, 2012. 
The appendices contain the following: Appendix A presents further diagrams of the results, indicating those within each language group. Appendix B includes omitted results and proofs.

\section{A Figures of Results}
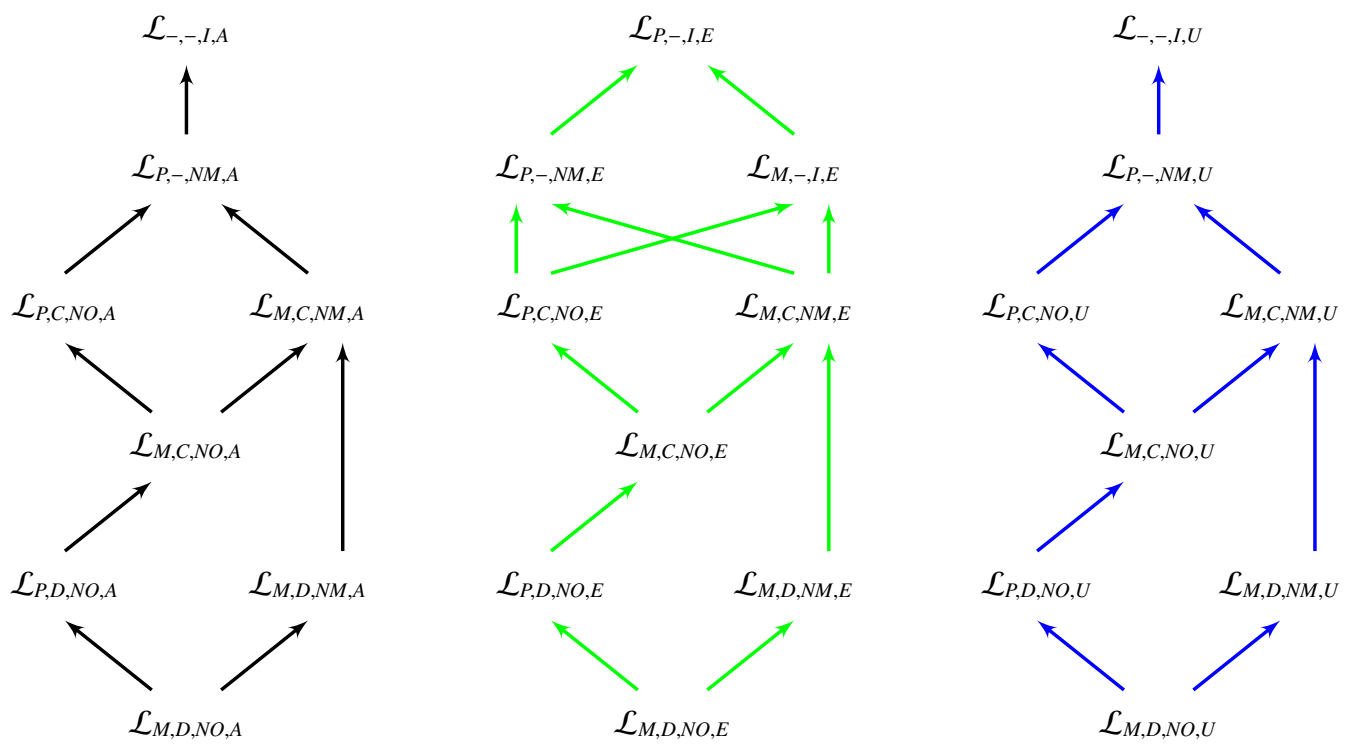

Fig. 3. Relations within Each Symmetric Language Groups

Figure 3 shows the relations within: the asymmetric languages as known from prior works; the exchange (green) languages as formalised in Section 5; and unification (blue) languages as formalised in Section 6. Arrows show increased expressive power. Black arrows and equalities are from prior work, green from Section 5, and blue from Section 6. The lack of an arrow indicates no possible encoding in either direction (e.g. between $\mathcal{L}_{P, C, N O, E}$ and $\mathcal{L}_{M, C, N M, E}$ ). Transitive relations over greater distances where another language fits in between are omitted (e.g. $\mathcal{L}_{P, D, N O, E}$ to $\mathcal{L}_{P, C, N O, E}$ ). Note that as mentioned in Section 3, such transitive relations are ensured here by the encodings presented here, although they do not hold in general for valid encodings. Within the exchange languages only, this indicates the similarity to the asymmetric languages at the bottom of the diagram, with differences appearing at the top with polyadicity and intensionality having different expressiveness relations under exchange. The relations within the unification language group clearly mirror those of the asymmetric languages.

The combined diagram illustrating the results all together is here shown in Figure 4 (this is a repeat of Figure 2 in Section 4). The following list details which results correspond to each arrow (an encoding result and a separation result) and equivalence (two encoding results). 


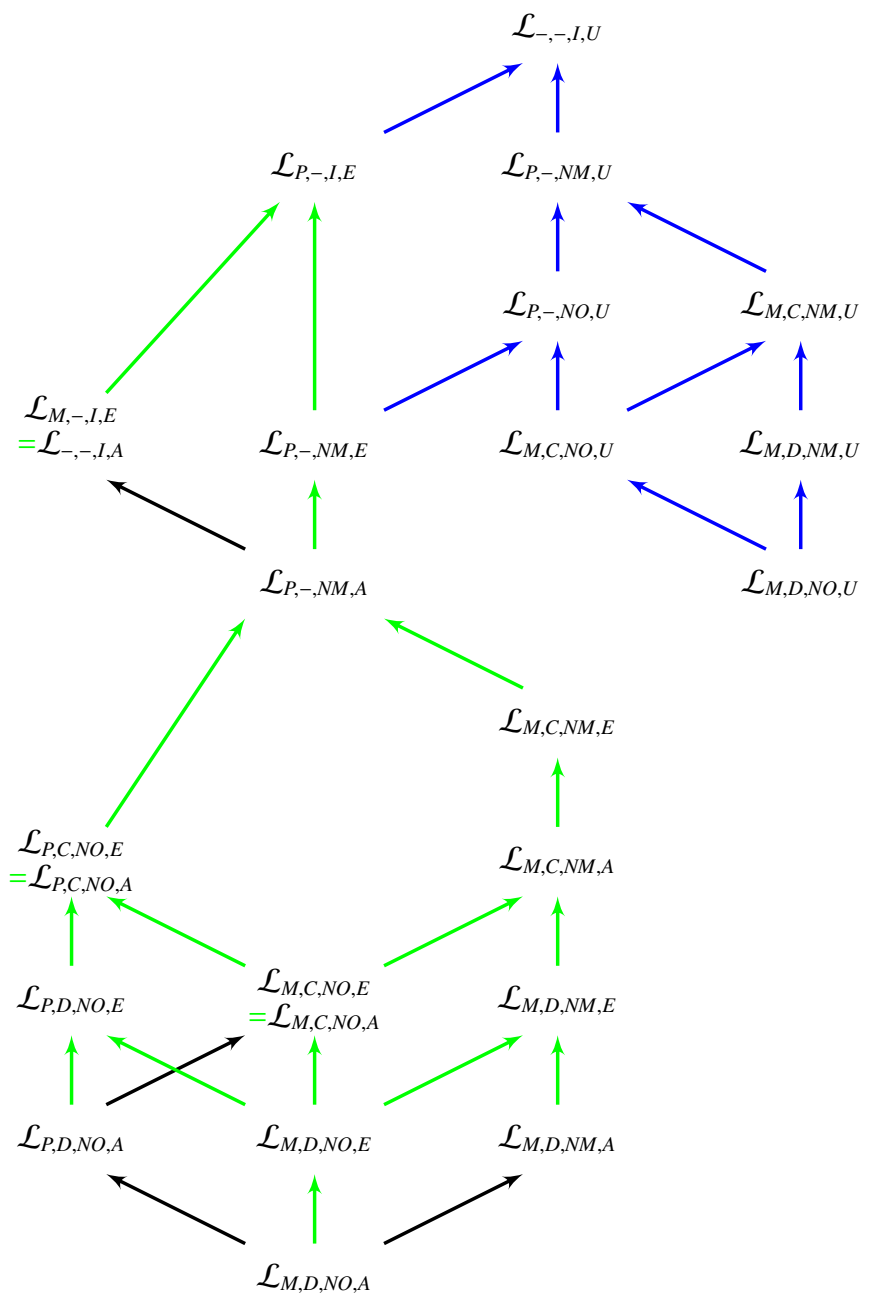

Fig. 4. Relations between all languages 
$\mathcal{L}_{M, D, N O, A} \rightarrow \mathcal{L}_{P, D, N O, A}$ The encoding and separation are both proved in [16].

$\mathcal{L}_{M, D, N O, A} \rightarrow \mathcal{L}_{M, D, N O, E}$ The encoding is via Proposition 4 and the separation result by adapting Theorem 1.

$\mathcal{L}_{M, D, N O, A} \rightarrow \mathcal{L}_{M, D, N M, A}$ The encoding and separation are both proved in [16].

$\mathcal{L}_{P, D, N O, A} \rightarrow \mathcal{L}_{P, D, N O, E}$ The encoding is via Proposition 4 and the separation result by Theorem 8.

$\mathcal{L}_{P, D, N O, A} \rightarrow \mathcal{L}_{M, C, N O, A}$ The encoding and separation are both proved in [16].

$\mathcal{L}_{M, D, N O, E} \rightarrow \mathcal{L}_{P, D, N O, E}$ The encoding is via Proposition 4 and the separation result by Theorem 7.

$\mathcal{L}_{M, D, N O, E} \rightarrow \mathcal{L}_{M, C, N O, A}$ The encoding is via Theorem 4 and the separation by application of Proposition 2.

$\mathcal{L}_{M, D, N O, E} \rightarrow \mathcal{L}_{M, D, N M, E}$ The encoding is via Proposition 4 and the separation result by application of Proposition 2.

$\mathcal{L}_{M, D, N M, A} \rightarrow \mathcal{L}_{M, D, N M, E}$ The encoding is via Proposition 4 and the separation result by adapting Theorem 1.

$\mathcal{L}_{M, C, N O, E}=\mathcal{L}_{M, C, N O, A}$ From left to right by Theorem 3 and from right to left by Proposition 4.

$\mathcal{L}_{P, D, N O, E} \rightarrow \mathcal{L}_{P, C, N O, A}$ The encoding is via Theorem 5 and the separation result by application of Proposition 2.

$\mathcal{L}_{M, C, N O, E} \rightarrow \mathcal{L}_{P, C, N O, E}$ The encoding is via Proposition 4 and the separation result by of Theorem 7.

$\mathcal{L}_{M, C, N O, E} \rightarrow \mathcal{L}_{M, C, N M, A}$ The encoding is by Theorem 3 and Proposition 4, and the separation by application of Proposition 2.

$\mathcal{L}_{M, D, N M, E} \rightarrow \mathcal{L}_{M, C, N M, A}$ The encoding is by an adaptation of Theorem 4 and the separation result by application of Proposition 2.

$\mathcal{L}_{P, C, N O, E}=\mathcal{L}_{P, C, N O, A}$ From left to right by Theorem 6 and from right to left by Proposition 4.

$\mathcal{L}_{P, C, N O, E} \rightarrow \mathcal{L}_{P,-, N M, A}$ The encoding is via Proposition 4 and the separation result by application of Proposition 2.

$\mathcal{L}_{M, C, N M, A} \rightarrow \mathcal{L}_{M, C, N M, E}$ The encoding is by Proposition 4 and the separation result by Theorem 1.

$\mathcal{L}_{M, C, N M, E} \rightarrow \mathcal{L}_{P,-, N M, A}$ The encoding is by Proposition 4 and the separation result by application of Proposition 2.

$\mathcal{L}_{P,-N M, A} \rightarrow \mathcal{L}_{-,-, I, A}$ The encoding and separation are both proved in [12].

$\mathcal{L}_{P,-, N M, A} \rightarrow \mathcal{L}_{P,-, N M, E}$ The encoding is via Proposition 4 and the separation result by Theorem 11.

$\mathcal{L}_{M, D, N O, U} \rightarrow \mathcal{L}_{M, C, N O, U}$ The encoding is via Proposition 4 and the separation result by application of Proposition 2.

$\mathcal{L}_{M, D, N O, U} \rightarrow \mathcal{L}_{M, D, N M, U}$ The encoding is via Proposition 4 and the separation result by Theorem 14.

$\mathcal{L}_{M,-, I, E}=\mathcal{L}_{-,-, I, A}$ From left to right by an adaptation of Theorem 4 and from right to left by Proposition 4.

$\mathcal{L}_{M,-, I, E} \rightarrow \mathcal{L}_{P,-, I, E}$ The encoding is via Proposition 4 and the separation result by an adaptation of Theorem 10.

$\mathcal{L}_{P,-, N M, E} \rightarrow \mathcal{L}_{P,-, I, E}$ The encoding is via Proposition 4 and the separation result by Theorem 10 . 
$\mathcal{L}_{P,-, N M, E} \rightarrow \mathcal{L}_{P,-, N O, U}$ The encoding is by Theorem 15 and the separation result by Theorem 12.

$\mathcal{L}_{M, C, N O, U} \rightarrow \mathcal{L}_{P,-, N O, U}$ The encoding is via Proposition 4 and the separation result by application of Proposition 2.

$\mathcal{L}_{M, C, N O, U} \rightarrow \mathcal{L}_{M, C, N M, U}$ The encoding is via Proposition 4 and the separation result by an adaptation of Theorem 14.

$\mathcal{L}_{M, D, N M, U} \rightarrow \mathcal{L}_{M, C, N M, U}$ The encoding is via Proposition 4 and the separation result by application of Proposition 2.

$\mathcal{L}_{P,-, N O, U} \rightarrow \mathcal{L}_{P,-, N M, U}$ The encoding is via Proposition 4 and the separation result by an adaptation of Theorem 14.

$\mathcal{L}_{M, C, N M, U} \rightarrow \mathcal{L}_{P,-, N M, U}$ The encoding is via Proposition 4 and the separation result by application of Proposition 2.

$\mathcal{L}_{P,-, I, E} \rightarrow \mathcal{L}_{-,-, I, U}$ The encoding is proved by Theorem 16 and the separation result by Theorem 12.

$\mathcal{L}_{P,-, N M, U} \rightarrow \mathcal{L}_{-,-, I, U}$ The encoding is by Proposition 4 and the separation result by using the techniques of Theorem 10. 


\section{B Omitted Proofs}

This appendix contains results and proofs omitted from the main paper.

\section{B.1 Proofs From Section 3}

The following are recalled for use in the proofs of this paper.

Proposition 5 (Proposition 5.5 from [18]). Let $\llbracket \cdot \rrbracket$ be a valid encoding; then, $S \mapsto$ implies that $\llbracket S \rrbracket \mapsto$.

Proposition 6 (Proposition 5.6 from [18]). Let $\llbracket \cdot \rrbracket$ be a valid encoding; then for every set of names $N$, it holds that $C_{1}^{N}\left(\cdot \cdot_{1}, \cdot_{2}\right)$ has both its holes at top-level.

Proposition 7 (Proposition 5.7 from [18]). Let $\llbracket \cdot \rrbracket$ be a valid encoding; if there exist two processes $S_{1}$ and $S_{2}$ such that $S_{1} \mid S_{2} \Downarrow$, with $S_{i} \Downarrow$ and $S_{i} \mapsto$ for $i=1$, 2, then $\llbracket S_{1} \rrbracket \mid \llbracket S_{2} \rrbracket \longmapsto$.

Proof (Proof of Proposition 1). By replacing $P$ with $\mathbf{0}$ and $Q$ with $\sqrt{ }$ the proof can easily by obtained by adapting that of Proposition 7 above.

Proof (Proof of Proposition 4). The encoding is trivial and $\llbracket P \rrbracket \stackrel{\text { def }}{=} P$ for all forms. The proof is then straightforward and ensured by definition of the poly-match rule for the base reduction. For a detailed example of the proof technique see Theorem 3.

\section{B.2 Proofs From Section 5}

Proof (Proof of Theorem 1). The proof is by contradiction. Assume there exists a valid encoding $\llbracket \cdot \rrbracket$ from $\mathcal{L}_{M, C, N M, E}$ into $\mathcal{L}_{M, C, N M, A}$. Consider the processes $P_{1}=n(\lambda x)$.if $x=$ $b$ then $\sqrt{ }$ and $P_{2}=\bar{n}\langle a\rangle . P_{2}^{\prime}$ where $P_{2}^{\prime}$ does not succeed. Since $P_{1} \mid P_{2} \longmapsto$ by validity of the encoding and Proposition $1 \llbracket P_{1} \mid P_{2} \rrbracket \longmapsto$ and this must be between some $R_{1}=\overline{m_{1}}\left\langle c_{1}\right\rangle \cdot R_{1}^{\prime}$ and $R_{2}$ for some $m_{1}$ and $c_{1}$ and $R_{1}^{\prime}$ and $R_{2}$. (This can be obtained by induction over the derivation tree for $\llbracket P_{1} \mid P_{2} \rrbracket \longmapsto R$.) Observe that $R_{1} \mid R_{2}$ cannot be a parallel component of either $\llbracket P_{1} \rrbracket$ or $\llbracket P_{2} \rrbracket$ since then by Proposition 5 either $P_{1}$ or $P_{2}$ would reduce and this is not the case.

If $R_{1}$ is a top-level component of $\llbracket P_{1} \rrbracket$ then $R_{2}$ must be top-level in $\llbracket P_{2} \rrbracket$. Now consider $R_{2}$.

1. If $R_{2}$ is of the form $m_{1}(\lambda z) \cdot R_{2}^{\prime}$ for some $z$ and $R_{2}^{\prime}$ then consider the process $Q=$ $n(\ulcorner a\urcorner) \cdot Q^{\prime}$. Clearly $P_{2} \mid Q \longmapsto$ and so $\llbracket P_{2} \mid Q \rrbracket \longmapsto$ by Proposition 1 . If this is from some $S$ that is top level in $\llbracket Q \rrbracket$ then consider the substitution $\sigma=\{a / b\}$. Since $P_{2} \mid \sigma Q \not$ then $R_{2} \mid \sigma^{\prime} S \longmapsto$ by Proposition 5, where $\sigma^{\prime}$ is defined by $\llbracket \sigma Q \rrbracket \cong \sigma^{\prime} \llbracket Q \rrbracket$ and name invariance of $\llbracket \cdot \rrbracket$. If the $R_{2} \mid \sigma^{\prime} S \longmapsto$ then this can only be due to a renaming of $m_{1}$ in $\llbracket Q \rrbracket$ by the poly-match rule. However, it can be shown that this renaming must also apply in $\sigma^{\prime} R_{1}$ and so $\sigma^{\prime} R_{1} \mid R_{2} \longmapsto$ yielding contradiction since $\sigma P_{1} \mid P_{2} \longmapsto$. 
2. If $R_{2}$ is of the form $m_{1}\left(\left\ulcorner c_{1}\right\urcorner\right) \cdot R_{2}^{\prime}$ then consider the process $Q_{1}=\bar{n}\langle b\rangle \cdot Q_{1}^{\prime}$. Clearly $P_{1} \mid Q_{1} \longmapsto$ and $P_{2} \mid Q_{1} \Downarrow$ and so $\llbracket P_{1} \mid Q_{1} \rrbracket$ must also by Proposition 7 and success sensitiveness. By reasoning as in the above case and exploiting substitutions such as $\sigma$ it follows that $c_{1}$ must not depend upon $a$ or $b$ since this would contradict operational correspondence, name invariance, or Propositions 5 or 7. Consider $Q_{2}=n\left(\ulcorner a) \cdot Q_{2}^{\prime}\right.$, clearly $P_{2} \mid Q_{2} \longmapsto$ and so $\llbracket P_{2} \mid Q_{2} \rrbracket \longmapsto$ by Proposition 1 . If 【 $Q_{2} \rrbracket$ interacts with $R_{2}$ then this must not depend upon $a$ and so $\llbracket \rho Q_{2} \mid P_{2} \rrbracket \longmapsto$ which contradicts Proposition 5 since $\rho Q_{2} \mid P_{2} \longmapsto$. Otherwise if $\llbracket Q_{2} \rrbracket$ interacts with some other top-level $S$ in $\llbracket P_{2} \rrbracket$ then this can be shown to yield divergence as in Theorem 7.1 (sub-case 2) of [12].

If $R_{1}$ is a top-level component of « $P_{2} \rrbracket$ then $R_{2}$ must arise from $\llbracket P_{1} \rrbracket$. If $R_{2}$ is of the form $m_{1}\left(\left\ulcorner c_{1}\right\urcorner\right) \cdot R_{2}^{\prime}$ then the same approach as case 2 above can be used to show contradiction. Therefore $R_{2}$ must be of the form $m_{1}(\lambda z) \cdot R_{2}^{\prime}$ for some $z$ and $R_{2}^{\prime}$. Now consider the process $Q=n(\ulcorner a\urcorner)$. $Q^{\prime}$. Since $Q \mid P_{2} \longmapsto$ it follows that $\llbracket Q \mid P_{2} \rrbracket \longmapsto$ by Proposition 1. If the reduction $\llbracket Q \mid P_{2} \rrbracket \longmapsto$ does not involve $R_{1}$ then obtain contradiction via divergence as in Theorem 7.1 (sub-case 2) of [12]. Therefore, $\llbracket Q \|$ must include some top-level input $m_{1}(p)$ such that $\operatorname{Match}\left(p, c_{1}\right)$ is defined. Now consider $p$. If $p$ is of the form $\lambda w$ then the reduction $\llbracket Q \mid P_{2} \rrbracket \longmapsto$ cannot depend upon the name $a$ in $Q$ and contradiction is yielded as in case 1 above. Finally, if $p$ is of the form $\left\ulcorner c_{1}\right\urcorner$ then $m_{1}\left(\left\ulcorner c_{1}\right\urcorner\right)$ cannot bind any names, and so there cannot be a restricted channel used for further interaction as in the encoding from $\mathcal{L}_{M, D, N O, E}$ into $\mathcal{L}_{M, C, N O, A}$. This can be exploited to show that since there is no restricted channel, the processes $P_{3}=\bar{n}\langle\lambda x\rangle$.if $x=c$ then $\Omega$ and $P_{4}=n(b) . P_{4}^{\prime}$ (where $\Omega$ is a divergent process) can yield contradiction by one of: $\llbracket P_{1} \mid P_{4} \rrbracket \longmapsto$ when $P_{1} \mid P_{4} \longmapsto$, or $\llbracket P_{3} \mid P_{2} \rrbracket \longmapsto$ when $P_{3} \mid P_{2} \not \longmapsto$, or choosing $\rho$ such that $\llbracket \rho\left(P_{1}\left|P_{2}\right| P_{3} \mid P_{4}\right) \rrbracket$ both succeeds and diverges, when $\rho\left(P_{1}\left|P_{2}\right| P_{3} \mid P_{4}\right)$ can only succeed or diverge but not both.

Proof (Proof of Theorem 2). To show there is no valid encoding from $\mathcal{L}_{M, D, N M, E}$ into $\mathcal{L}_{M, C, N O, E}$ it suffices to consider the processes $P_{1}=(\lambda x) . \mathbf{0}$ and $P_{2}=(\ulcorner a\urcorner) \cdot \sqrt{ }$ and $P_{3}=$ $\left.\langle a\rangle .\left({ }^{\prime}\right\urcorner\right) .0$. Observe that $P_{3}$ reduces with both $P_{1}$ and $P_{2}$, reporting success with $P_{2}$. However, take $\sigma=\{b / a, a / b\}$ and now $\sigma P_{3}$ does not reduce with $P_{2}$. Any encoding must either: allow $\llbracket P_{2} \mid \sigma P_{3} \rrbracket$ to reduce; or not allow $\llbracket P_{2} \mid P_{3} \rrbracket$ to reduce; both of which contradict Proposition 5. In the other direction the technique of Theorem 4.5 of [16] can be used.

The following lemmas are used for the proof of the Theorem 3 below.

Lemma 1. If $P \equiv Q$ then $\llbracket P \rrbracket \equiv \llbracket Q \rrbracket$. Conversely, if $\llbracket P \rrbracket \equiv Q$ then $Q=\llbracket P^{\prime} \rrbracket$ for some $P^{\prime} \equiv P$.

Proof. Straightforward, the only non-trivial part is when renaming has occurred.

Lemma 2. Given $\mathcal{L}_{M, C, N O, E}$ action $P$ and co-action $Q$ then $\llbracket P \rrbracket \mid \llbracket Q \rrbracket \longmapsto$ if and only if $P \mid Q \longmapsto$.

Proof. Both parts can be proved by induction on the height of the proof tree for the judgments $\llbracket P \mid Q \rrbracket \longmapsto$ and $P \mid Q \longmapsto$. The base case is ensured by the poly-match rule when $P$ is of the form $n(\lambda x) \cdot P^{\prime}$ or $\bar{n}\langle\lambda x\rangle . P^{\prime}$. Note that Lemma 1 is used to ensure structural congruence. 
Lemma 3. The translation $\llbracket \cdot \rrbracket$ from $\mathcal{L}_{M, C, N O, E}$ into $\mathcal{L}_{M, C, N O, A}$ preserves and reflects reductions. That is:

1. If $P \longmapsto P^{\prime}$ then $\llbracket P \rrbracket \longmapsto \llbracket P^{\prime} \rrbracket$;

2. if $\llbracket P \rrbracket \longmapsto Q$ then $Q=\llbracket P^{\prime} \rrbracket$ for some $P^{\prime}$ such that $P \longmapsto P^{\prime}$.

Proof. Both parts can be proved by straightforward induction on the judgments $P \longmapsto$ $P^{\prime}$ and $\llbracket P \rrbracket \longmapsto Q$, respectively. In both cases, the base step is the most interesting and follows from Lemma 2, for the second case the step $Q \longmapsto Q^{\prime}$ is ensured by the definition of the translation and match rule. The inductive cases where the last rule used is a structural one then rely on Lemma 1.

Proof (Proof of Theorem 3). Compositionality and name invariance hold by construction. Operational correspondence (with structural equivalence in the place of $\cong$ ) and divergence reflection follow from Lemma 3. Success sensitiveness can be proved as follows: $P \Downarrow$ means that there exists $P^{\prime}$ and $k \geq 0$ such that $P \longmapsto^{k} P^{\prime} \equiv P^{\prime \prime} \mid \sqrt{ }$; by exploiting Lemma $3 k$ times and Lemma 1 obtain that $\llbracket P \rrbracket \longmapsto^{3 k} \llbracket P^{\prime} \rrbracket \equiv \llbracket P^{\prime \prime} \rrbracket \mid \sqrt{ }$, i.e. that $\llbracket P \rrbracket \Downarrow$. The converse implication can be proved similarly.

Observe that the same proof technique can be used for Theorems $4,5, \& 6$ by adapting the supporting lemmas where required.

Proof (Proof of Theorem 7). The proof is by contradiction. Assume there exists a valid encoding $\llbracket \cdot \rrbracket$ from $\mathcal{L}_{P, D, N O, E}$ into $\mathcal{L}_{M, D, N O, E}$. Consider the processes $P_{1}=(\lambda x, b)$.if $x=$ $a$ then $(\lambda x) \cdot \sqrt{ }$ and $P_{2}=(\lambda y, d) .0$ and $P_{3}=\langle c, \lambda x\rangle$.if $x=b$ then $\langle b\rangle$. $\sqrt{ }$ and $P_{4}=$ $\langle a, \lambda y\rangle$.0. Since $P_{1}\left|P_{2}\right| P_{3} \mid P_{4} \Downarrow$ it follows that $\llbracket P_{1}\left|P_{2}\right| P_{3} \mid P_{4} \rrbracket \Downarrow$. However, it can be shown that either $\llbracket P_{1}\left|P_{2}\right| P_{3} \mid P_{4} \rrbracket \mapsto$ or $\llbracket P_{1}\left|P_{2}\right| P_{3} \mid P_{4} \rrbracket \Downarrow$, both of which yield contradiction.

Proof (Proof of Theorem 8). The proof is by contradiction. Assume there is a valid encoding of $\mathcal{L}_{P, D, N O, E}$ into $\mathcal{L}_{P, D, N O, A}$. The key to the proof is to consider the processes $P_{1}=$ if $x=a$ then $(\lambda z) \cdot \sqrt{ }$ and $P_{2}=$ if $y=d$ then $\langle z\rangle . \mathbf{0}$. Clearly neither $(\lambda x, b) \cdot P_{1} \mid\langle a, \lambda y\rangle . P_{2}$ nor $(\lambda x, d) . \mathbf{0} \mid\langle c, \lambda y\rangle . \mathbf{0}$ report success and by validity of the encoding their encodings do not either. Conclude by showing that the process $\left((\lambda x, b) . P_{1} \mid\langle a, \lambda y\rangle . P_{2}\right) \mid((\lambda x, d) . \mathbf{0} \mid\langle c, \lambda y\rangle . \mathbf{0})$ does not report success while showing that its encoding $\llbracket\left((\lambda x, b) . P_{1} \mid\langle a, \lambda y\rangle . P_{2}\right) \mid((\lambda x, d) . \mathbf{0} \mid\langle c, \lambda y\rangle . \mathbf{0}) \rrbracket$ does.

Proof (Proof of Theorem 9). This can be proved in the same manner as Theorem 3 by adapting the supporting lemmas where required.

Proof (Proof of Theorem 10). The proof technique is the same as Theorem 7.1 of [12]. The technique exploits the arity $k$ of the reduction between the encoded processes $P_{0}=$ $(\lambda x) .\langle m\rangle . \mathbf{0}$ and $P_{1}=\langle a\rangle . \mathbf{0}$ to show that another encoded process $P_{2}=\left\langle a_{1} \bullet \ldots \bullet\right.$ $\left.a_{k+2}\right\rangle . \mathbf{0}$ must either: interact with arity $<k+2$ (and then fail to match some name $a_{i}$ and contradict operational correspondence); or results in divergence.

Proof (Proof of Theorem 11). The proof is by contradiction, assume there exists a valid encoding $\llbracket \cdot \rrbracket$. Consider the following processes $P_{1}=\left(a,\left\ulcorner b^{\urcorner}\right) .\left(\left\ulcorner m^{\urcorner}\right) \cdot \sqrt{ }\right.\right.$ and $P_{2}=$ 
$\langle\ulcorner\urcorner, d\rangle .\langle m\rangle . \mathbf{0}$ where $a$ and $b$ and $c$ and $d$ are pairwise distinct names. Consider the substitutions $\sigma_{1}=\{a / c\}$ and $\sigma_{2}=\{b / d\}$. Observe that none of $P_{1} \mid P_{2}$ or $\sigma_{1}\left(P_{1} \mid P_{2}\right)$ or $\sigma_{2}\left(P_{1} \mid P_{2}\right)$ reduce or report success, and thus their encodings do not either. However, $\sigma_{1}\left(\sigma_{2}\left(P_{1} \mid P_{2}\right)\right)$ does reduce and report success, and thus there exists $\sigma_{1}^{\prime}$ and $\sigma_{2}^{\prime}$ such that $R=\sigma_{1}^{\prime}\left(\sigma_{2}^{\prime}\left(\llbracket P_{1} \mid P_{2} \rrbracket\right)\right)$ does reduce and report success. Now consider the names matched in the first reduction of $R \longmapsto R^{\prime}$. Clearly if the names are not in the union of the ranges of $\sigma_{1}^{\prime}$ and $\sigma_{2}^{\prime}$ then $\sigma_{1}^{\prime}\left(\llbracket P_{1} \mid P_{2} \rrbracket\right)$ or $\sigma_{2}^{\prime}\left(\llbracket P_{1} \mid P_{2} \rrbracket\right)$ or $\llbracket P_{1} \mid P_{2} \rrbracket$ would reduce, but this would contradict a validity of the encoding. Now by exploiting the process $P_{3}=\left\langle\ulcorner c, \lambda z\rangle .\langle m, d\rangle . \mathbf{0}\right.$ it can be shown that either $\sigma_{1}^{\prime}\left(\sigma_{2}^{\prime}\left(\llbracket P_{1} \mid P_{3} \rrbracket\right)\right) \mapsto$ or $\sigma_{1}^{\prime}\left(\sigma_{2}^{\prime}\left(\llbracket P_{1}\left|P_{2}\right| P_{3} \rrbracket\right)\right)$ is divergent, both of which yield contradiction.

\section{B.3 Proofs From Section 6}

The following lemma is used for the proof of the theorem below.

Lemma 4. Given a language $\mathcal{L}_{1}$ that it non-unification, then for all $\mathcal{L}_{1}$ processes $S$ such that $S \mapsto$, it holds that $S \mid S \mapsto$.

Proof. By contradiction. Assume that $S \mid S \longmapsto$ and consider how this reduction occurred. If $S \longmapsto S^{\prime}$ this contradicts $S \mapsto$.

It follows that the reduction must be of some $a(\widetilde{p}) \cdot S_{1}\left|\bar{a}\langle\widetilde{q}\rangle . S_{2} \longmapsto \sigma S_{1}\right| \rho S_{2}$ where $\operatorname{Match}(\widetilde{p} ; \widetilde{q})=(\sigma, \rho)$ and $S \mid S \equiv(\widetilde{n n})\left(a(\widetilde{p}) . S_{1}\left|\bar{a}\langle\widetilde{q}\rangle . S_{2}\right| R\right)$ for some $R$ (the $a$ 's are omitted in the dataspace-based languages). It is straightforward to show that both $a(\widetilde{p}) . S_{1}$ and $\bar{a}\langle\widetilde{q}\rangle . S_{2}$ must be contained within $S$, and that for the reduction to occur it must be possible for $S \longmapsto$ yielding contradiction.

Proof (Proof of Theorem 12). Observe that in all the unification languages the self recognising process $S$ is such that $S \mapsto$ and $S \Downarrow$, however $S \mid S \longmapsto$ and $S \mid S \Downarrow$. Conclude by Proposition 3 and Lemma 4 .

Proof (Proof of Theorem 13). By contradiction. Assume there exists a valid encoding $\llbracket \cdot \rrbracket$ from $\mathcal{L}_{M, D, N O, \delta}$ where $\delta \neq U$ into $\mathcal{L}_{M, D, N O, U}$. Consider the $\mathcal{L}_{M, D, N O, \delta}$ processes $P_{1}=(\lambda x) \cdot \sqrt{ }$ and $P_{2}=\langle a\rangle \cdot \sqrt{ }$. Since neither of $P_{1}$ or $P_{2}$ reduce or report success it follows that their encodings do not. Since $P_{1} \mid P_{2}$ does reduce and report success it follows that $\llbracket P_{1} \mid P_{2} \rrbracket$ also reduces and reports success. By considering the context $C_{1}^{\mathcal{X}}\left(\llbracket\left[P_{1} \rrbracket, \llbracket P_{2} \rrbracket\right)\right.$ the reduction must be due to some $(p) . S^{\prime}$ and some $(b) . T^{\prime}$ for some $p$ and $b$ and $S^{\prime}$ and $T^{\prime}$. By validity of the encoding it must be that $(b) . T^{\prime}$ is part of $\llbracket P_{i} \rrbracket$ for $i \in\{1,2\}$. Observe that by definition of the poly-unify rule $(b) \cdot T^{\prime} \mid(b) \cdot T^{\prime}$ reduces. Now the context $C_{\mid}^{\mathcal{X}}\left(\llbracket P_{i} \rrbracket, \llbracket P_{i} \rrbracket\right)$ can be shown to reduce (and report success), however this contradicts that $P_{i} \mid P_{i} \mapsto$.

Proof (Proof of Theorem 14). The proof is by contradiction, assume that there exists a valid encoding $\llbracket \cdot \rrbracket$ from $\mathcal{L}_{M, D, N M, U}$ into $\mathcal{L}_{M, D, N O, U}$. Now consider the process $P_{1}=$ $(\ulcorner a\urcorner) . \sqrt{ }$. Clearly $P_{1}$ does not reduce or report success and so it's encoding does not either. However, $P_{1} \mid P_{1}$ does reduce and report success, and so $\llbracket P_{1} \mid P_{1} \rrbracket$ must also. By definition of the poly-unify rule the reduction $\llbracket P_{1} \mid P_{1} \rrbracket$ must be between an action (b). $S^{\prime}$ and another action, now consider this other action: 
- If the action is $(\lambda z) . S^{\prime \prime}$ then it must arise from the encoding $\llbracket P_{1} \rrbracket$. It follows via reasoning over the structure of $\llbracket P_{1} \rrbracket$ that $\llbracket P_{1} \rrbracket$ must be able to reduce, but this contradicts the validity of the encoding.

- If the action is $(b) . S^{\prime \prime}$ then it can be shown that $b$ must be determined by $a$ and the encoding (otherwise $P_{2}=(c) .0$ with $\{a, b, c\}$ pairwise distinct would yield that [ $P_{1} \mid P_{2} \rrbracket$ reduces while $P_{1} \mid P_{2}$ does not). Now consider the processes $P_{3}=$ $(\lambda z)$.if $a=z$ then $\sqrt{ }$ and $P_{4}=(a) .0$. Since $P_{1} \mid P_{4}$ reduces and reports success it follows that the encoding $\llbracket P_{1} \mid P_{4} \rrbracket$ must do also.

- Now if $\llbracket\left[P_{4} \rrbracket\right.$ interacts via only $(b) . T$ then since $P_{3} \mid P_{4}$ reduce then $\llbracket P_{3} \rrbracket$ must be able to interact with $(b) . T$. However, then $\llbracket P_{1} \mid P_{3} \rrbracket$ would reduce, which contradicts the validity of encoding.

- If $\llbracket P_{4} \rrbracket$ interacts via only the form $(\lambda q) . T$ then it follows that $\llbracket P_{4} \mid P_{4} \rrbracket$ does not reduce while $P_{4} \mid P_{4}$ does, yielding contradiction.

- Therefore $\llbracket P_{4} \rrbracket$ must have multiple forms of interaction, which can in turn be used to show that the encoding is contradictory via either operational correspondence or divergence reflection.

Proof (Proof of Theorem 15). This can be proved in the same manner as Theorem 3 by adapting the supporting lemmas where required.

Proof (Proof of Theorem 16). This can be proved in the same manner as Theorem 3 by adapting the supporting lemmas where required. 